Article

\title{
LC-MS Lipidomics: Exploiting a Simple High-Throughput Method for the Comprehensive Extraction of Lipids in a Ruminant Fat Dose-Response Study
}

\author{
Benjamin Jenkins ${ }^{1} \mathbb{D}$, Martin Ronis ${ }^{2}$ and Albert Koulman ${ }^{1, *} \mathbb{D}$ \\ 1 NIHR BRC Core Metabolomics and Lipidomics Laboratory, University of Cambridge, Pathology Building \\ Level 4, Addenbrooke's Hospital, Cambridge CB2 0QQ, UK; bjj25@medschl.cam.ac.uk \\ 2 College of Medicine, Department of Pharmacology \& Experimental Therapeutics, Louisiana State University \\ Health Sciences Centre, 1901 Perdido Str., New Orleans, LA 70112, USA; mronis@lsuhsc.edu \\ * Correspondence: AK675@medschl.cam.ac.uk
}

Received: 22 June 2020; Accepted: 16 July 2020; Published: 17 July 2020

\begin{abstract}
Typical lipidomics methods incorporate a liquid-liquid extraction with LC-MS quantitation; however, the classic sample extraction methods are not high-throughput and do not perform well at extracting the full range of lipids especially, the relatively polar species (e.g., acyl-carnitines and glycosphingolipids). In this manuscript, we present a novel sample extraction protocol, which produces a single phase supernatant suitable for high-throughput applications that offers greater performance in extracting lipids across the full spectrum of species. We applied this lipidomics pipeline to a ruminant fat dose-response study to initially compare and validate the different extraction protocols but also to investigate complex lipid biomarkers of ruminant fat intake (adjoining onto simple odd chain fatty acid correlations). We have found 100 lipids species with a strong correlation with ruminant fat intake. This novel sample extraction along with the LC-MS pipeline have shown to be sensitive, robust and hugely informative ( $>450$ lipids species semi-quantified): with a sample preparation throughput of over 100 tissue samples per day and an estimated 1000 biological fluid samples per day. Thus, this work facilitating both the epidemiological involvement of ruminant fat, research into odd chain lipids and also streamlining the field of lipidomics (both by sample preparation methods and data presentation).
\end{abstract}

Keywords: odd chain lipids; lipid profiling; Folch; protein precipitation; sample preparation; relative lipid composition (Mol\%)

\section{Introduction}

Lipids are generally understood as a class of molecules that have a high solubility in organic solvents and typically contain or originate from fatty acids. Although, lipids may be commonly derived, research has shown that there is a huge variety both structurally and functionally (potentially $>40,000$ [1]); where they play a vital role in energy production and storage [2,3], regulation and signalling [4,5], provide structure and support and membrane formation [6]. Lipids are now emerging as biomarkers of dietary/nutritional intakes [7] as well as indicators of pathophysiological status [8-11]. As a reaction of lipid-pathophysiological involvements, the field of lipidomics has emerged as a discipline that examines and quantifies a large proportion of the lipids present in a given sample set.

Lipidomics requires an effective isolation protocol that comprehensively extracts lipids from the sample as well as an analytical method that allows their identification and quantitation. The typical analyte isolation protocols (with/without minor adaptations) that are often used in the literature include 
three different liquid-liquid extractions: Folch and colleagues [12] (cited $>65,000$ times), Bligh and Dyer [13] (cited $>52,000$ times) or Matyash and colleagues [14] (cited $>1000$ times). Although these extraction protocols are heavily cited and do result in adequate results, there are several caveats with their use. Firstly, there is the need to perform duplicate extraction in non-fluid samples to ensure optimal recovery of the lipid analytes. This is extremely time consuming, especially for the Folch and the Bligh and Dyer methods. Secondly, there are reasonable concerns that using a biphasic extraction (producing immiscible aqueous and organic phases) may result in a loss of relatively polar lipids (e.g., acyl-carnitines and gangliosides) into the disposed aqueous fractions (consisting of mostly methanol and water in these extraction protocols: Folch and the Bligh and Dyer). There are publications that use a single phase extraction protocol but they do not appear to solve the problem of extracting the relatively more polar lipids since a mixture of methanol, chloroform and tert-butyl methyl ether were used $[15,16]$.

The technique overwhelmingly used tor analysing the lipidome is mass spectrometry hyphenated with chromatography (LC-MS) due to its sensitivity and selectivity; furthermore by using a high-resolution accurate mass instrument (e.g., Orbitrap or Time-of-Flight instruments), a huge number of analytes can be analysed simultaneously. Reversed phase chromatography is the predominant chromatographic technique employed to separate the analytes before entering the mass spectrometer to determine their structure and concentration. Variants of a liquid chromatography method utilising a C18-column with a water and acetonitrile mix for the weak eluting mobile phase and acetonitrile and propan-2-ol for the strong eluting mobile phase are the most commonly used [17-22]. These reversed phase C18-column methods both separate lipid based on their lipid class assignment (i.e., either phosphatidylcholines or phosphatidyethanolamines head group) and their fatty-acyl composition (i.e., chain length and degree of unsaturation) with some degree of isomeric separation.

In this study, we present a lipidomics pipeline that including a novel analyte isolation protocol utilising a single phase, which results in a comprehensive lipid extraction suitable for a full range of lipid polarities (from polar to non-polar lipids species). This lipidomics method was tested, validated and then applied in a rat model investigating ruminant fat biomarkers via a beef tallow dose response dietary investigation.

\section{Results}

This lipidomics LC-MS method incorporating both of the described sample preparation protocols: protein precipitation (chloroform: methanol: acetone, 7:3:4) and Folch liquid-liquid (chloroform: methanol: water, 7:3:4), were utilised for the quantitation of lipids in liver samples from Sprague-Dawley rats who received one of four experimental diets overfed at $17 \%$ above matched growth.

A comparison of the two sample preparation methods on the extraction of the stable isotope-labelled internal standards are shown in the figure below (see Figure 1). A comparison on the samples' endogenous individual lipid classes are shown in the Supplementary Materials (see Supplementary Figure S1 and Table S1). 


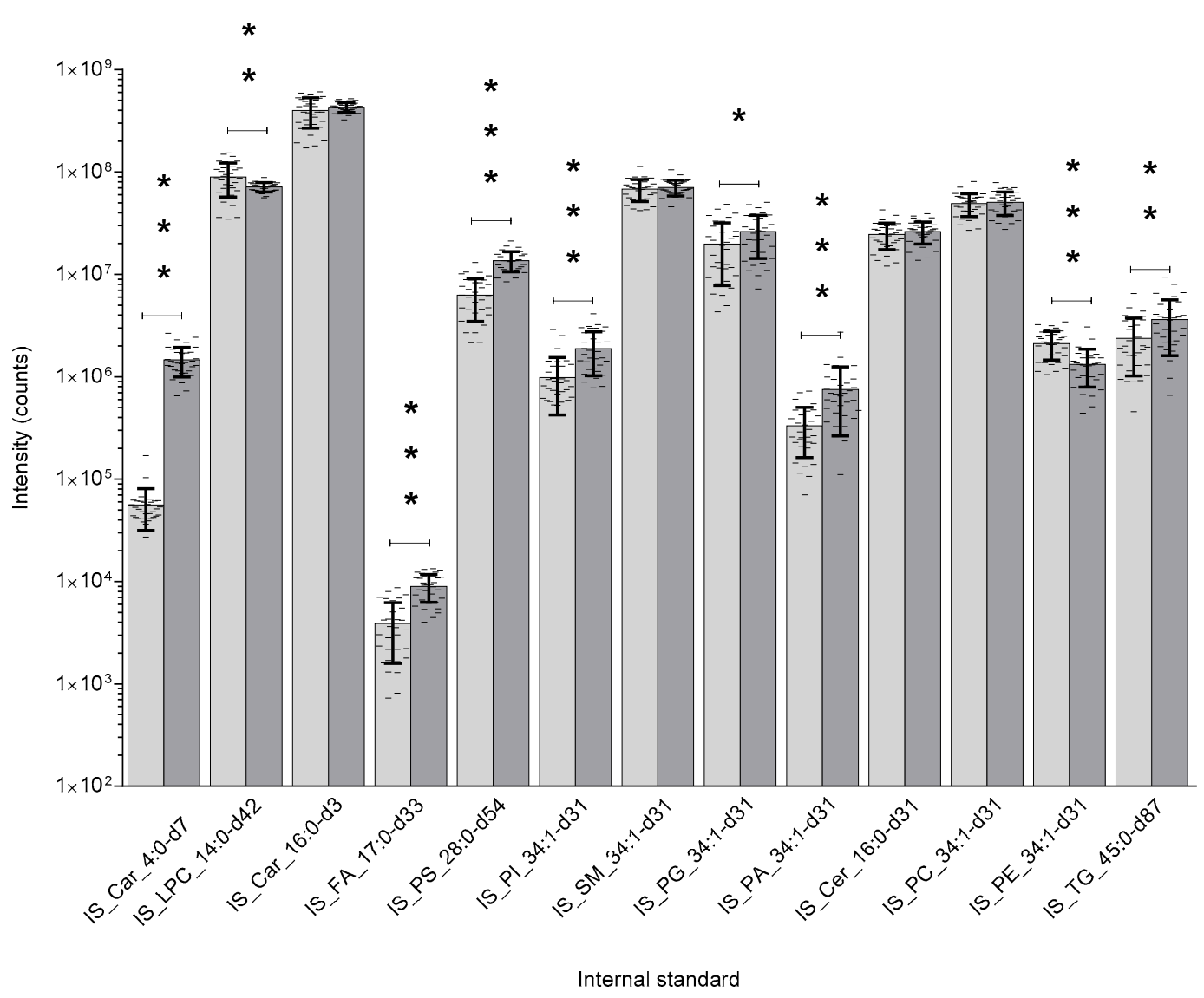

Figure 1. This figure shows the comparison between the two lipid extraction techniques regarding their extraction efficiency of the stable isotope internal standards from the rat liver samples (Folch liquid-liquid extraction: chloroform: methanol: water, 7:3:4 $\square$, and Protein precipitation liquid extraction: chloroform: methanol: acetone, $\sim 7: 3: 4 \square$ ). $n=34$ rat liver samples per extraction method. The intensity of the internal standards were measured by liquid chromatography with mass spectrometry. The significance of the difference between the two extraction protocols are shown by the p-value star system; where $p \leq 0.05$ was considered statistically significant $\left({ }^{*} p<0.05,{ }^{* *} p<0.01,{ }^{* * *} p<0.001\right)$. Error bars represent \pm standard deviation. Lipid internal standard include: Butyryl-d7-L-carnitine (abbreviated to IS_Car_4:0-d7), N-tetradecylphosphocholine-d42 (abbreviated to IS_LPC_14:0-d42), hexadecanoyl-L-carnitine-d3 (abbreviated to IS_Car_16:0-d3), heptadecanoic-d33 acid (abbreviated to IS_FA_17:0-d33), 1,2-dimyristoyl-d54-sn-glycero-3-[phospho-L-serine] (abbreviated to IS_PS_28:0-d54), 1-palmitoyl-d31-2-oleoyl-sn-glycero-3-phosphoinositol (abbreviated to IS_PI_34:1-d31), N-palmitoyl-d31-D-erythro-sphingosylphosphorylcholine (abbreviated to IS_SM_34:1-d31), 1-palmitoyl-d31-2-oleoyl-sn-glycero-3-[phospho-rac-(1-glycerol)] (abbreviated to IS_PG_34:1-d31), 1-palmitoyl-d31-2-oleoyl-sn-glycero-3-phosphate (abbreviated to IS_PA_34:1-d31), N-palmitoyl-d31-D-erythro-sphingosine (abbreviated to IS_Cer_16:0-d31), 1-palmitoyl-d31-2-oleoyl-sn-glycero-3-phosphocholine (abbreviated to IS_PC_34:1-d31), 1-palmitoyl-d31-2-oleoyl-sn-glycero-3-phosphoethanolamine (abbreviated to IS_PE_34:1-d31), glyceryl tri(pentadecanoate-d29) (abbreviated to IS_TG_45:0-d87).

As shown the intensity of seven of the internal standards are statistically significantly higher in the protein precipitation protocol when compared to the Folch liquid-liquid protocol (between $\sim 30 \%$ to $\sim 2500 \%$ higher), whereas, only two of the internal standards were higher in the Folch liquid-liquid protocol (between $\sim 20 \%$ and $\sim 37 \%$ higher). Additionally, there is far less variation in the protein precipitation liquid extraction protocol: 10 out of 13 internal standards had $\sim 2 \%$ to $72 \%$ less variation in their coefficient of variation $(\mathrm{CV})$. The protein precipitation liquid extraction protocol 
has also been shown to produce a significantly higher detection of the samples' endogenous lipids, both producing a higher total number of lipid detected (Folch-LLE: 455 lipid species, PPLE: 472 lipid species) and a statistically significantly higher total intensity for twelve of the sixteen lipid classes detected (see Supplementary Figure S1 and Table S1). Taken as a whole, the protein precipitation protocol (chloroform: methanol: acetone, 7:3:4) showed a greater extraction capability across the full lipid hydro-philicity/phobicity range and across the internal standards. Additionally, the high throughput of the protein precipitation protocol allows for over 100 tissue samples to be extracted per day (including dissection, weighing and tissue extraction ready for LC-MS analysis), whereas, the Folch liquid-liquid protocol could take up to three- to four-times longer due to the necessity of duplicate extractions and the delicacy of liquid-liquid phase separation. The throughput of the protein precipitation protocol (chloroform: methanol: acetone, $~ 7: 3: 4$ ) on fluid samples allows an estimated $\sim 1000$ biological fluid sample extractions per day (including aliquoting, sample extraction ready for LC-MS analysis) when utilising basic laboratory fluid handling equipment/robots (throughput data not shown here).

The liver lipid concentration $(\mathrm{nM} / \mathrm{mg})$ for each experimental diet group of rats are shown in the table below (see Table 1), along with the correlation (trendline equation, slope significance, $\mathrm{R}^{2}$ and successive change across the groups) of the measured lipid concentration with the percentage composition of ruminant fat (beef tallow) in each experimental diet. An $R^{2}$ threshold of 0.75 , slope significance $p$-value $<0.05$ and successive increase/decrease were set to establish if there was a strong correlation between the lipid concentration and the ruminant fat composition. 
Table 1. This table shows the liver lipid concentrations from the Sprague-Dawley rats who received one of four experimental diets ( $\mathrm{n}=8-9$ per group). Diet $1: 50 \%$ corn oil, 16.4\% MCT oil and 3.6\% beef tallow; Diet 2: 35\% corn oil, 28.7\% MCT oil and 6.3\% beef tallow; Diet 3: 20\% corn oil, 41.0\% MCT oil and 9.0\% beef tallow; Diet 4: $5 \%$ corn oil, 53.3\% MCT oil and 11.7\% beef tallow. MCT: medium chain triglyceride oil. Lipid are shown in their shorthand notations with the number of carbons and unsaturated bonds in the fatty acid moiety separated by a colon; acyl-carnitines (Carn), ceramides (Cer), cardiolipins (CL), diacylglycerols (DG), gangliosides (GM1), hexosylceramides (Hex-Cer), lyso-phosphatidylcholines (LPC), lyso-phosphatidyethanolamines (LPE), lyso-phosphatidylinositols (LPI), lyso-cardiolipins (Lyso_CL), phosphatidic acids (PA), phosphatidylcholines (PC), phosphatidylethanolamines (PE), phosphatidylglycerol (PG), phosphatidylinositols (PI), phosphatidylserines (PS), sulfatides (S), sphingomyelins (SM), triacylglycerides (TG). Lipid concentrations (nM/mg) are shown as mean \pm standard deviation and were extracted via the protein precipitation liquid extraction protocol (chloroform: methanol: acetone, $7: 3: 4)$. Correlation between the ruminant fat composition of the diet and the lipid concentration are depicted by the trendline equation $\left({ }^{*}\right.$ denotes statistical significance of the slope: $p$-value $\left.<0.05\right)$ and coefficient of determination $\left(R^{2}\right)$. Lipid concentrations continually increasing/decreasing across the groups as the ruminant fat composition of the diet increase are emphasised in the 'Successive change across group' column. Lipids with a significant slope ( $p$-value $<0.05)$ with an $\mathrm{R}^{2}$ greater than 0.75 and successively increasing/decreasing are in bold and highlighted.

\begin{tabular}{|c|c|c|c|c|c|c|c|}
\hline & Diet 1 & Diet 2 & Diet 3 & Diet 4 & Trendline Equation & $\mathbf{R}^{2}$ & $\begin{array}{c}\text { Successive Chang } \\
\text { Across Groups }\end{array}$ \\
\hline Carn_(C00:0) & $99,100 \pm 19,200$ & $90,200 \pm 38,800$ & $126,000 \pm 29,000$ & $92,400 \pm 49,600$ & $y=581 x+97,500$ & 0.02 & \\
\hline Carn_(C02:0) & $10,400 \pm 4150$ & $10,700 \pm 3280$ & $12,900 \pm 6740$ & $11,700 \pm 5300$ & $y=226 x+9700$ & 0.49 & \\
\hline Carn_(C03:0) & $3430 \pm 1380$ & $3590 \pm 1820$ & $3760 \pm 2170$ & $3830 \pm 2240$ & $y=50.7 x+3260 *$ & 0.97 & Increasing \\
\hline Carn $(\mathrm{C} 03: 0-2 \mathrm{COOH})$ & $713 \pm 326$ & $436 \pm 299$ & $943 \pm 438$ & $700 \pm 604$ & $y=17.3 x+565$ & 0.08 & \\
\hline Carn_(C03:0-OH) & $\mathrm{ND} \pm \mathrm{ND}$ & $1.46 \pm 4.39$ & $2.1 \pm 5.94$ & $6.16 \pm 13$ & $y=0.87 x-4.59$ & 0.85 & \\
\hline Carn_(C03:1) & $108 \pm 64.2$ & $105 \pm 58.2$ & $101 \pm 75.6$ & $75.5 \pm 35.3$ & $y=-3.76 x+126$ & 0.78 & Decreasing \\
\hline Carn_(C04:0) & $1040 \pm 847$ & $675 \pm 342$ & $1560 \pm 659$ & $793 \pm 309$ & $y=5.33 x+976$ & 0.00 & \\
\hline Carn_(C04:0-OH) & $952 \pm 429$ & $702 \pm 503$ & $1730 \pm 944$ & $1230 \pm 619$ & $y=69 x+626$ & 0.30 & \\
\hline Carn_(C04:1) & $134 \pm 56$ & $102 \pm 94.4$ & $89.6 \pm 63.3$ & $104 \pm 78.4$ & $y=-3.79 x+136$ & 0.49 & \\
\hline Carn_(C05:0) & $490 \pm 534$ & $359 \pm 173$ & $857 \pm 577$ & $553 \pm 437$ & $y=25.4 x+370$ & 0.18 & \\
\hline Carn_(C05:1) & $319 \pm 125$ & $255 \pm 87.3$ & $317 \pm 205$ & $227 \pm 99.5$ & $y=-7.93 x+340$ & 0.36 & \\
\hline Carn_(C06:0) & $324 \pm 287$ & $183 \pm 61.5$ & $635 \pm 296$ & $234 \pm 123$ & $y=6.74 x+292$ & 0.01 & \\
\hline Carn_(C06:0-2COOH $)$ & $511 \pm 145$ & $362 \pm 124$ & $742 \pm 246$ & $477 \pm 213$ & $y=10.3 x+444$ & 0.05 & \\
\hline Carn_(C08:0) & $168 \pm 174$ & $107 \pm 66.6$ & $412 \pm 185$ & $186 \pm 215$ & $y=13.3 x+117$ & 0.12 & \\
\hline Carn_(C08:1) & $81.7 \pm 50.9$ & $102 \pm 56.5$ & $124 \pm 54.8$ & $88.3 \pm 62.7$ & $y=1.55 x+87.2$ & 0.08 & \\
\hline Carn_(C10:0-2COOH) & $353 \pm 178$ & $361 \pm 124$ & $378 \pm 146$ & $296 \pm 177$ & $y=-5.7 x+391$ & 0.31 & \\
\hline Carn_(C12:0) & $0.265 \pm 0.339$ & $0.147 \pm 0.353$ & $0.495 \pm 0.291$ & $0.412 \pm 0.402$ & $y=0.0292 x+0.106$ & 0.43 & \\
\hline Carn_(C14:0) & $11.1 \pm 6.52$ & $9.39 \pm 8.48$ & $14.8 \pm 5.36$ & $13 \pm 5.69$ & $y=0.411 x+8.92$ & 0.38 & \\
\hline Carn_(C15:0) & $4.33 \pm 2.64$ & $3.76 \pm 2.87$ & $4.88 \pm 1.52$ & $3.65 \pm 0.927$ & $\mathrm{y}=-0.0341 \mathrm{x}+4.42$ & 0.04 & \\
\hline Carn_(C16:0) & $618 \pm 339$ & $457 \pm 315$ & $739 \pm 411$ & $462 \pm 226$ & $y=-6.89 x+622$ & 0.03 & \\
\hline Carn_(C16:0-OH) & $1.2 \pm 1.56$ & $1.52 \pm 1.98$ & $1.61 \pm 1.35$ & $2.46 \pm 2.18$ & $y=0.143 x+0.601$ & 0.86 & Increasing \\
\hline Carn_(C16:2) & $9.89 \pm 7.09$ & $9.69 \pm 4.49$ & $7.61 \pm 4.01$ & $10.9 \pm 4.92$ & $y=0.0352 x+9.25$ & 0.01 & \\
\hline Carn_(C17:0) & $19.2 \pm 8.93$ & $13.9 \pm 6.7$ & $22.3 \pm 9.04$ & $18.4 \pm 7.41$ & $y=0.222 x+16.8$ & 0.05 & \\
\hline Carn_(C18:0) & $627 \pm 220$ & $541 \pm 170$ & $656 \pm 154$ & $620 \pm 221$ & $y=3.48 x+584$ & 0.06 & \\
\hline Carn_(C18:0-OH) & $5.47 \pm 3.31$ & $6.8 \pm 3.5$ & $5.41 \pm 4.18$ & $8.14 \pm 4.52$ & $y=0.245 x+4.58$ & 0.44 & \\
\hline
\end{tabular}


Table 1. Cont

\begin{tabular}{|c|c|c|c|c|c|c|c|}
\hline & Diet 1 & Diet 2 & Diet 3 & Diet 4 & Trendline Equation & $\mathbf{R}^{2}$ & $\begin{array}{l}\text { Successive Change } \\
\text { Across Groups }\end{array}$ \\
\hline Carn_(C18:1) & $1530 \pm 1090$ & $1230 \pm 931$ & $1900 \pm 958$ & $1130 \pm 449$ & $y=-19.6 x+1600$ & 0.04 & \\
\hline Carn (C18:2) & $824 \pm 754$ & $631 \pm 525$ & $374 \pm 157$ & $505 \pm 259$ & $y=-45 x+927$ & 0.67 & \\
\hline Carn_(C18:3) & $16.8 \pm 16.4$ & $14.7 \pm 13.3$ & $11.5 \pm 6.38$ & $14.8 \pm 5.16$ & $y=-0.341 x+17.1$ & 0.29 & \\
\hline Carn_(C20:0) & $43.1 \pm 38.9$ & $41.9 \pm 28.2$ & $32.5 \pm 20.4$ & $45.9 \pm 22.9$ & $y=-0.037 x+41.1$ & 0.00 & \\
\hline Carn_(C22:5) & $2.6 \pm 3.13$ & $2.34 \pm 2.81$ & $1.7 \pm 1.25$ & $1.66 \pm 1.06$ & $y=-0.128 x+3.06 *$ & 0.91 & Decreasing \\
\hline Cer_(32:1) & $5.17 \pm 1.26$ & $4.31 \pm 0.783$ & $5.34 \pm 1.78$ & $5.4 \pm 1.75$ & $y=0.0637 x+4.57$ & 0.19 & \\
\hline Cer_(33:1) & $2.29 \pm 1.92$ & $1.68 \pm 1.26$ & $1.48 \pm 0.544$ & $1.27 \pm 0.925$ & $y=-0.121 x+2.6 *$ & 0.92 & Decreasing \\
\hline Cer_(34:0) & $0.918 \pm 0.57$ & $1.19 \pm 1.13$ & $2.53 \pm 2.19$ & $1.93 \pm 1.51$ & $y=0.162 x+0.402$ & 0.60 & \\
\hline Cer_(34:1) & $1.49 \pm 0.581$ & $1.23 \pm 0.881$ & $1.79 \pm 1.01$ & $2.56 \pm 0.989$ & $y=0.14 x+0.699$ & 0.71 & \\
\hline Cer $(35: 0)$ & $\mathrm{ND} \pm \mathrm{ND}$ & $\mathrm{ND} \pm \mathrm{ND}$ & $0.0674 \pm 0.191$ & $\mathrm{ND} \pm \mathrm{ND}$ & & & \\
\hline Cer_(35:1) & $1.03 \pm 1.06$ & $1.61 \pm 1.06$ & $1.63 \pm 1.08$ & $2.16 \pm 1.45$ & $y=0.126 x+0.641 *$ & 0.91 & Increasing \\
\hline Cer_(36:0) & $7.26 \pm 2.79$ & $9.98 \pm 5.3$ & $10.5 \pm 4.07$ & $13.1 \pm 2.89$ & $y=0.668 x+5.1 *$ & 0.95 & Increasing \\
\hline Cer_(36:1) & $54.5 \pm 26.2$ & $55.4 \pm 10.4$ & $59.7 \pm 16.3$ & $71.7 \pm 24.5$ & $\mathrm{y}=2.07 \mathrm{x}+44.5$ & 0.83 & Increasing \\
\hline Cer_(36:2) & $6.59 \pm 3.55$ & $5.23 \pm 1.18$ & $5.94 \pm 2.23$ & $6.81 \pm 3.13$ & $y=0.0507 x+5.75$ & 0.06 & \\
\hline Cer $(37: 1)$ & $2.23 \pm 0.767$ & $1.9 \pm 0.723$ & $2.31 \pm 0.772$ & $4.23 \pm 1.09$ & $\mathrm{y}=0.237 \mathrm{x}+0.851$ & 0.61 & \\
\hline Cer_(37:2) & $\mathrm{ND} \pm \mathrm{ND}$ & $\mathrm{ND} \pm \mathrm{ND}$ & $0.066 \pm 0.187$ & $\mathrm{ND} \pm \mathrm{ND}$ & & & \\
\hline Cer_(38:0) & $2.14 \pm 1.19$ & $3.82 \pm 3.78$ & $2.1 \pm 0.715$ & $2.9 \pm 0.626$ & $y=0.0207 x+2.58$ & 0.01 & \\
\hline Cer $(38: 1)$ & $47.9 \pm 18.8$ & $34.1 \pm 7.21$ & $39.5 \pm 10.3$ & $56 \pm 13$ & $\mathrm{y}=1.1 \mathrm{x}+36$ & 0.16 & \\
\hline Cer_(38:2) & $6.76 \pm 3.87$ & $4.76 \pm 1.57$ & $4.7 \pm 0.967$ & $8.02 \pm 1.98$ & $y=0.138 x+5.01$ & 0.09 & \\
\hline Cer_(39:0) & $0.188 \pm 0.0814$ & $0.221 \pm 0.149$ & $0.243 \pm 0.153$ & $0.724 \pm 0.454$ & $y=0.0604 x-0.118$ & 0.68 & Increasing \\
\hline Cer_(39:1) & $10.5 \pm 3.44$ & $8.36 \pm 1.6$ & $10.6 \pm 1.62$ & $20.3 \pm 3.17$ & $y=1.17 x+3.48$ & 0.58 & \\
\hline Cer_(39:2) & $0.436 \pm 0.447$ & $0.332 \pm 0.333$ & $0.416 \pm 0.342$ & $1.65 \pm 0.755$ & $y=0.138 x-0.347$ & 0.58 & \\
\hline Cer_(40:0) & $3.71 \pm 0.85$ & $4.12 \pm 0.933$ & $4.74 \pm 1.44$ & $6.17 \pm 0.98$ & $y=0.296 x+2.42 *$ & 0.92 & Increasing \\
\hline Cer_(40:1) & $126 \pm 37.9$ & $116 \pm 18.7$ & $146 \pm 51$ & $227 \pm 39.1$ & $y=12.3 x+59.4$ & 0.73 & \\
\hline Cer_(40:2) & $65.5 \pm 24.7$ & $50.8 \pm 8.2$ & $63 \pm 13.4$ & $105 \pm 12.7$ & $y=4.84 x+34$ & 0.52 & \\
\hline Cer (41:0) & $2.95 \pm 0.547$ & $3.91 \pm 1.03$ & $4.94 \pm 1.2$ & $7.37 \pm 1.36$ & $y=0.529 x+0.744 *$ & 0.94 & Increasing \\
\hline Cer_(41:1) & $72.1 \pm 18.6$ & $89.7 \pm 20.4$ & $130 \pm 88.3$ & $210 \pm 48.4$ & $y=16.8 x-3.18 *$ & 0.91 & Increasing \\
\hline Cer_(41:2) & $51.4 \pm 19.1$ & $49.6 \pm 11.4$ & $63.3 \pm 6.75$ & $144 \pm 22.5$ & $y=10.8 x-5.52$ & 0.70 & \\
\hline Cer_(42:0) & $6.21 \pm 2.01$ & $8.11 \pm 2.54$ & $11.5 \pm 5.17$ & $12.2 \pm 3.76$ & $y=0.791 x+3.45 *$ & 0.95 & Increasing \\
\hline Cer_(42:1) & $310 \pm 63.3$ & $444 \pm 130$ & $624 \pm 539$ & $773 \pm 305$ & $y=58.1 x+93.2 *$ & 1.00 & Increasing \\
\hline Cer $(42: 2)$ & $575 \pm 161$ & $599 \pm 87.4$ & $721 \pm 120$ & $1180 \pm 187$ & $y=71.7 x+220$ & 0.79 & Increasing \\
\hline Cer_(42:3) & $105 \pm 40.2$ & $92 \pm 19.5$ & $103 \pm 26.1$ & $151 \pm 16.5$ & $y=5.52 x+70.5$ & 0.54 & \\
\hline Cer_(43:0) & $1.31 \pm 0.425$ & $2.05 \pm 0.699$ & $2.43 \pm 0.806$ & $3.07 \pm 0.982$ & $y=0.21 x+0.611 *$ & 0.99 & Increasing \\
\hline Cer_(43:1) & $131 \pm 31.2$ & $218 \pm 62.9$ & $253 \pm 87.4$ & $396 \pm 111$ & $y=30.7 x+14.3 *$ & 0.94 & Increasing \\
\hline Cer_(43:2) & $42.9 \pm 7.62$ & $58.9 \pm 11.9$ & $64.2 \pm 12.8$ & $115 \pm 17.2$ & $y=8.21 x+7.46$ & 0.84 & Increasing \\
\hline Cer_(44:1) & $16.7 \pm 4.31$ & $28.5 \pm 8.34$ & $38.9 \pm 26.1$ & $43 \pm 14.8$ & $y=3.31 x+6.47^{*}$ & 0.96 & Increasing \\
\hline Cer $(44: 2)$ & $0.908 \pm 0.271$ & $1.19 \pm 0.288$ & $1.72 \pm 1.08$ & $2.08 \pm 0.644$ & $y=0.15 x+0.328 *$ & 0.99 & Increasing \\
\hline Cer_(45:1) & $0.46 \pm 0.215$ & $1.19 \pm 0.531$ & $1.32 \pm 0.618$ & $1.58 \pm 0.746$ & $y=0.129 x+0.149$ & 0.88 & Increasing \\
\hline
\end{tabular}


Table 1. Cont

\begin{tabular}{|c|c|c|c|c|c|c|c|}
\hline & Diet 1 & Diet 2 & Diet 3 & Diet 4 & Trendline Equation & $\mathbf{R}^{2}$ & $\begin{array}{l}\text { Successive Change } \\
\text { Across Groups }\end{array}$ \\
\hline Cer_(45:2) & $\mathrm{ND} \pm \mathrm{ND}$ & $0.00348 \pm 0.0104$ & $0.00503 \pm 0.0142$ & $\mathrm{ND} \pm \mathrm{ND}$ & $y=0.000574 x-0.000137$ & 1.00 & \\
\hline Cer_(46:1) & $0.0023 \pm 0.0069$ & $0.0192 \pm 0.0266$ & $0.0409 \pm 0.0568$ & $0.0568 \pm 0.0824$ & $y=0.00686 x-0.0227 *$ & 1.00 & Increasing \\
\hline Cer_(46:2) & $0.83 \pm 0.187$ & $1.53 \pm 0.439$ & $1.57 \pm 0.516$ & $1.79 \pm 0.679$ & $y=0.108 x+0.603$ & 0.82 & Increasing \\
\hline CL_(66:02) & $8.65 \pm 6.02$ & $22.3 \pm 17.1$ & $32.3 \pm 17.1$ & $37.7 \pm 31.3$ & $y=3.6 x-2.29 *$ & 0.97 & Increasing \\
\hline CL_(66:03) & $15.8 \pm 10.1$ & $23.6 \pm 16.4$ & $24.2 \pm 13.2$ & $17.3 \pm 8.8$ & $y=0.189 x+18.8$ & 0.02 & \\
\hline CL_(66:04) & $15.5 \pm 10.7$ & $21.8 \pm 10.4$ & $29.7 \pm 16.7$ & $27 \pm 15.3$ & $y=1.57 x+11.5$ & 0.76 & \\
\hline CL_(66:05) & $40.5 \pm 20$ & $39.1 \pm 10.7$ & $18.5 \pm 10.3$ & $1 \pm 0.911$ & $y=-5.15 x+64.2 *$ & 0.92 & Decreasing \\
\hline CL_(66:06) & $66.1 \pm 37$ & $67.9 \pm 26.4$ & $65.1 \pm 38.1$ & $22.6 \pm 13.2$ & $y=-4.94 x+93.2$ & 0.62 & \\
\hline CL_(67:02) & $4.6 \pm 5.88$ & $15.8 \pm 13.3$ & $11.3 \pm 6.02$ & $10.1 \pm 10$ & $y=0.444 x+7.05$ & 0.11 & \\
\hline CL_(67:03) & $13.4 \pm 9.85$ & $22.4 \pm 14$ & $14.1 \pm 6.36$ & $5.97 \pm 4.69$ & $y=-1.13 x+22.6$ & 0.35 & \\
\hline CL_(67:05) & $0.848 \pm 1.74$ & $1.4 \pm 2.57$ & $2.58 \pm 2.02$ & $3.16 \pm 1.62$ & $y=0.301 x-0.303 *$ & 0.98 & Increasing \\
\hline CL_(68:00) & $0.484 \pm 0.502$ & $0.898 \pm 1.1$ & $0.998 \pm 1.12$ & $0.519 \pm 1.01$ & $y=0.00759 x+0.667$ & 0.01 & \\
\hline CL_(68:01) & $105 \pm 91.1$ & $260 \pm 222$ & $190 \pm 107$ & $142 \pm 163$ & $y=1.52 x+163$ & 0.01 & \\
\hline CL_(68:02) & $469 \pm 385$ & $1010 \pm 799$ & $664 \pm 355$ & $434 \pm 480$ & $y=-16.7 x+772$ & 0.05 & \\
\hline CL_(68:03) & $631 \pm 421$ & $1030 \pm 670$ & $560 \pm 261$ & $237 \pm 199$ & $y=-61.2 x+1080$ & 0.43 & \\
\hline CL_(68:04) & $367 \pm 179$ & $439 \pm 197$ & $294 \pm 113$ & $184 \pm 112$ & $y=-25.7 x+518$ & 0.68 & \\
\hline CL_(69:04) & $40.4 \pm 31.9$ & $66.9 \pm 41.7$ & $56.3 \pm 27.3$ & $28.8 \pm 18.6$ & $y=-1.68 x+61$ & 0.12 & \\
\hline CL_(69:05) & $64.8 \pm 37.5$ & $67.7 \pm 29.7$ & $51.2 \pm 20.4$ & $31.1 \pm 15.2$ & $y=-4.36 x+87$ & 0.83 & \\
\hline CL_(69:06) & $49.7 \pm 32.3$ & $49.7 \pm 20.5$ & $29.3 \pm 11$ & $25.1 \pm 10.8$ & $y=-3.49 x+65.1$ & 0.86 & \\
\hline CL_(69:07) & $0.245 \pm 0.736$ & $1.06 \pm 0.942$ & $1.33 \pm 1.17$ & $0.685 \pm 0.79$ & $y=0.0589 x+0.38$ & 0.19 & \\
\hline CL_(70:01) & $1.45 \pm 1.87$ & $6.38 \pm 7.09$ & $4.32 \pm 2.94$ & $3.91 \pm 5.53$ & $y=0.197 x+2.51$ & 0.12 & \\
\hline CL_(70:02) & $61.2 \pm 59.7$ & $102 \pm 90.1$ & $54.6 \pm 31.2$ & $33.4 \pm 40.7$ & $y=-4.84 x+99.9$ & 0.35 & \\
\hline CL_(70:03) & $470 \pm 379$ & $796 \pm 600$ & $559 \pm 271$ & $340 \pm 303$ & $y=-23.2 x+719$ & 0.18 & \\
\hline CL_(70:04) & $1270 \pm 814$ & $2010 \pm 1300$ & $1510 \pm 656$ & $950 \pm 761$ & $y=-54.1 x+1850$ & 0.18 & \\
\hline CL_(70:05) & $1770 \pm 889$ & $2210 \pm 1060$ & $1670 \pm 641$ & $1200 \pm 780$ & $y=-83.3 x+2350$ & 0.49 & \\
\hline CL_(70:06) & $1280 \pm 629$ & $1570 \pm 595$ & $1560 \pm 688$ & $1440 \pm 897$ & $y=17.4 x+1330$ & 0.20 & \\
\hline CL_(70:07) & $882 \pm 480$ & $1110 \pm 399$ & $1210 \pm 657$ & $971 \pm 551$ & $y=13.6 x+939$ & 0.11 & \\
\hline CL_(70:08) & $256 \pm 199$ & $245 \pm 108$ & $147 \pm 56.7$ & $89.7 \pm 52.6$ & $y=-22.1 x+354 *$ & 0.93 & Decreasing \\
\hline CL_(70:09) & $25.8 \pm 18.3$ & $27.1 \pm 13.6$ & $17.7 \pm 8.62$ & $14.1 \pm 7.15$ & $y=-1.65 x+33.8$ & 0.83 & \\
\hline CL_(71:02) & $0.744 \pm 1.36$ & $2.59 \pm 3$ & $1.41 \pm 1.38$ & $0.451 \pm 0.714$ & $y=-0.0763 x+1.88$ & 0.08 & \\
\hline CL_(71:03) & $7.35 \pm 7.45$ & $20.2 \pm 18.2$ & $18.2 \pm 11$ & $9.9 \pm 8.38$ & $y=0.209 x+12.3$ & 0.01 & \\
\hline CL_(71:04) & $19.5 \pm 12.7$ & $51.1 \pm 39.5$ & $65.7 \pm 41.9$ & $43 \pm 33$ & $y=3.15 x+20.7$ & 0.32 & \\
\hline CL_(71:05) & $56.9 \pm 35$ & $116 \pm 76.6$ & $130 \pm 56.9$ & $107 \pm 69.8$ & $y=6.09 x+55.9$ & 0.44 & \\
\hline CL_(71:06) & $106 \pm 65.4$ & $164 \pm 78.1$ & $192 \pm 74.8$ & $164 \pm 88.4$ & $y=7.48 x+99.3$ & 0.52 & \\
\hline CL_(71:07) & $76.3 \pm 55.8$ & $104 \pm 37.6$ & $120 \pm 42.8$ & $101 \pm 54.8$ & $y=3.34 x+74.8$ & 0.41 & \\
\hline CL_(71:08) & $23.9 \pm 25.3$ & $17.1 \pm 6.59$ & $5.98 \pm 4.09$ & $0.44 \pm 0.928$ & $y=-3.02 x+34.9 *$ & 0.98 & Decreasing \\
\hline CL_(72:01) & $0.251 \pm 0.441$ & $1.36 \pm 1.4$ & $0.758 \pm 0.648$ & $0.825 \pm 1.56$ & $y=0.0415 x+0.481$ & 0.10 & \\
\hline
\end{tabular}


Table 1. Cont

\begin{tabular}{|c|c|c|c|c|c|c|c|}
\hline & Diet 1 & Diet 2 & Diet 3 & Diet 4 & Trendline Equation & $\mathbf{R}^{2}$ & $\begin{array}{l}\text { Successive Change } \\
\text { Across Groups }\end{array}$ \\
\hline CL_(72:02) & $0.874 \pm 1.29$ & $10.3 \pm 12.3$ & $4.1 \pm 2.73$ & $4.24 \pm 7.52$ & $y=0.144 x+3.77$ & 0.02 & \\
\hline CL $(72: 03)$ & $22.6 \pm 21.7$ & $55.2 \pm 54.7$ & $23.4 \pm 12.2$ & $17.9 \pm 25$ & $y=-1.7 x+42.8$ & 0.12 & \\
\hline CL_(72:04) & $246 \pm 202$ & $374 \pm 291$ & $228 \pm 110$ & $237 \pm 229$ & $y=-6.41 x+320$ & 0.11 & \\
\hline CL_(72:05) & $2310 \pm 1300$ & $3190 \pm 1750$ & $2650 \pm 1080$ & $2000 \pm 1500$ & $y=-54.4 x+2950$ & 0.14 & \\
\hline CL_(72:06) & $10,800 \pm 5640$ & $12,200 \pm 4950$ & $9770 \pm 3660$ & $5820 \pm 3810$ & $y=-643 x+14,600$ & 0.67 & \\
\hline CL_(72:07) & $28,500 \pm 15,500$ & $25,400 \pm 6760$ & $17,100 \pm 6180$ & $7210 \pm 4430$ & $y=-2670 x+40,000 *$ & 0.96 & Decreasing \\
\hline CL_(72:08) & $31,100 \pm 17,500$ & $24,200 \pm 5090$ & $13,800 \pm 4820$ & $4420 \pm 2860$ & $y=-3350 x+44,000 *$ & 0.99 & Decreasing \\
\hline CL_(72:09) & $1280 \pm 774$ & $1150 \pm 378$ & $798 \pm 339$ & $456 \pm 284$ & $y=-105 x+1720 *$ & 0.97 & Decreasing \\
\hline CL_(72:10) & $66.9 \pm 45.2$ & $62.8 \pm 21.4$ & $62 \pm 40.5$ & $70.8 \pm 37.4$ & $y=0.404 x+62.5$ & 0.12 & \\
\hline$C_{-} L_{-}(74: 06)$ & $1080 \pm 745$ & $930 \pm 448$ & $489 \pm 203$ & $395 \pm 366$ & $y=-92.4 x+1430 *$ & 0.94 & Decreasing \\
\hline CL_(74:07) & $4550 \pm 2900$ & $3350 \pm 1190$ & $2000 \pm 712$ & $1650 \pm 1280$ & $y=-372 x+5740 *$ & 0.95 & Decreasing \\
\hline CL $(74: 08)$ & $8200 \pm 4930$ & $6210 \pm 1800$ & $4510 \pm 1460$ & $3490 \pm 2360$ & $y=-586 x+10,100 *$ & 0.98 & Decreasing \\
\hline CL_(74:09) & $7180 \pm 3960$ & $6480 \pm 1830$ & $5090 \pm 1530$ & $3460 \pm 2210$ & $y=-465 x+9110 *$ & 0.97 & Decreasing \\
\hline CL_(74:10) & $3980 \pm 2390$ & $3050 \pm 652$ & $1860 \pm 595$ & $1030 \pm 673$ & $y=-372 x+5320$ * & 1.00 & Decreasing \\
\hline CL_(74:11) & $1240 \pm 707$ & $784 \pm 177$ & $454 \pm 136$ & $284 \pm 181$ & $y=-118 x+1600 *$ & 0.96 & Decreasing \\
\hline CL_(76:09) & $802 \pm 495$ & $560 \pm 202$ & $416 \pm 152$ & $633 \pm 566$ & $y=-24.1 x+787$ & 0.27 & \\
\hline CL_(76:10) & $1300 \pm 724$ & $974 \pm 335$ & $723 \pm 242$ & $960 \pm 768$ & $y=-47.1 x+1350$ & 0.48 & \\
\hline CL $(76: 11)$ & $1580 \pm 894$ & $1140 \pm 399$ & $678 \pm 217$ & $710 \pm 530$ & $y=-114 x+1900$ & 0.87 & \\
\hline CL_(76:12) & $1200 \pm 704$ & $925 \pm 337$ & $513 \pm 163$ & $441 \pm 328$ & $y=-99.6 x+1530 *$ & 0.94 & Decreasing \\
\hline DG_(32:0) & $194 \pm 108$ & $64.5 \pm 72.4$ & $75.8 \pm 122$ & $99.9 \pm 108$ & $y=-10 x+185$ & 0.35 & \\
\hline DG_(34:0) & $84.3 \pm 167$ & $48.3 \pm 145$ & $\mathrm{ND} \pm \mathrm{ND}$ & $\mathrm{ND} \pm \mathrm{ND}$ & $y=-13.3 x+132$ & 1.00 & \\
\hline DG_(34:1) & $6950 \pm 4570$ & $3290 \pm 1420$ & $3300 \pm 1600$ & $4690 \pm 4060$ & $y=-251 x+6480$ & 0.26 & \\
\hline GM1 $(34: 0)$ & $1.47 \pm 1.39$ & $5.46 \pm 4.45$ & $4.11 \pm 4.43$ & $3.26 \pm 2.36$ & $\mathrm{y}=0.149 \mathrm{x}+2.44$ & 0.10 & \\
\hline GM1_(34:1) & $4.89 \pm 3.76$ & $15.3 \pm 11.1$ & $10.6 \pm 9.26$ & $9.27 \pm 6.24$ & $y=0.313 x+7.62$ & 0.06 & \\
\hline GM1_(34:1-OH) & $0.054 \pm 0.0451$ & $0.0988 \pm 0.0944$ & $0.0491 \pm 0.0531$ & $0.0338 \pm 0.0588$ & $y=-0.00409 x+0.0902$ & 0.26 & \\
\hline GM1_(36:0) & $\mathrm{ND} \pm \mathrm{ND}$ & $0.0285 \pm 0.0603$ & $\mathrm{ND} \pm \mathrm{ND}$ & $\mathrm{ND} \pm \mathrm{ND}$ & & & \\
\hline GM1_(36:1) & $\mathrm{ND} \pm \mathrm{ND}$ & $0.157 \pm 0.356$ & $0.125 \pm 0.158$ & $0.152 \pm 0.285$ & $y=-0.000926 x+0.153$ & 0.02 & \\
\hline Hex-Cer_(32:0) & $0.578 \pm 0.44$ & $0.383 \pm 0.558$ & $0.199 \pm 0.291$ & $0.332 \pm 0.324$ & $y=-0.0341 x+0.634$ & 0.57 & \\
\hline Hex-Cer_(32:1) & $\mathrm{ND} \pm \mathrm{ND}$ & $0.0486 \pm 0.104$ & $0.0269 \pm 0.076$ & $0.0396 \pm 0.112$ & $y=-0.00167 x+0.0534$ & 0.17 & \\
\hline Hex-Cer_(34:0-OH) & $0.0882 \pm 0.264$ & $0.0548 \pm 0.164$ & $0.0611 \pm 0.173$ & $\mathrm{ND} \pm \mathrm{ND}$ & $y=-0.00502 x+0.0997$ & 0.58 & \\
\hline Hex-Cer_(34:1) & $0.565 \pm 0.791$ & $1.27 \pm 1.2$ & $1.74 \pm 1.87$ & $4.68 \pm 1.85$ & $y=0.475 x-1.57$ & 0.84 & Increasing \\
\hline Hex-Cer_(34:1-OH) & $0.295 \pm 0.719$ & $\mathrm{ND} \pm \mathrm{ND}$ & $0.0372 \pm 0.105$ & $0.208 \pm 0.587$ & $y=-0.016 x+0.31$ & 0.25 & \\
\hline Hex-Cer_(34:2) & $0.32 \pm 0.418$ & $0.318 \pm 0.598$ & $\mathrm{ND} \pm \mathrm{ND}$ & $0.424 \pm 1.2$ & $y=0.0138 x+0.255$ & 0.88 & \\
\hline Hex-Cer_(34:2-OH) & $0.614 \pm 0.37$ & $0.518 \pm 0.375$ & $0.39 \pm 0.512$ & $0.306 \pm 0.488$ & $y=-0.039 x+0.755 *$ & 0.99 & Decreasing \\
\hline Hex-Cer_(35:0) & $0.0425 \pm 0.127$ & $\mathrm{ND} \pm \mathrm{ND}$ & $0.663 \pm 0.389$ & $1.37 \pm 1.56$ & $y=0.157 x-0.579$ & 0.95 & \\
\hline Hex-Cer_(35:1) & $0.551 \pm 0.625$ & $0.649 \pm 0.567$ & $1.03 \pm 0.58$ & $1.66 \pm 1.57$ & $y=0.137 x-0.0781 *$ & 0.91 & Increasing \\
\hline Hex-Cer (36:0-OH) & $2.03 \pm 1.06$ & $2.58 \pm 2.01$ & $3.51 \pm 1.07$ & $4.75 \pm 2.01$ & $y=0.337 x+0.642 *$ & 0.97 & Increasing \\
\hline Hex-Cer_(36:1) & $0.235 \pm 0.413$ & $\mathrm{ND} \pm \mathrm{ND}$ & $0.688 \pm 0.661$ & $0.264 \pm 0.491$ & $y=0.0151 x+0.274$ & 0.06 & \\
\hline
\end{tabular}


Table 1. Cont

\begin{tabular}{|c|c|c|c|c|c|c|c|}
\hline & Diet 1 & Diet 2 & Diet 3 & Diet 4 & Trendline Equation & $\mathbf{R}^{2}$ & $\begin{array}{l}\text { Successive Change } \\
\text { Across Groups }\end{array}$ \\
\hline Hex-Cer_(36:2) & $\mathrm{ND} \pm \mathrm{ND}$ & $0.0816 \pm 0.245$ & $0.289 \pm 0.574$ & $0.638 \pm 0.942$ & $y=0.103 x-0.591$ & 0.98 & \\
\hline Hex-Cer $(37: 0)$ & $\mathrm{ND} \pm \mathrm{ND}$ & $0.0835 \pm 0.167$ & $0.0639 \pm 0.181$ & $0.151 \pm 0.295$ & $y=0.0125 x-0.013$ & 0.55 & \\
\hline Hex-Cer_(37:0-OH) & $9.56 \pm 9.47$ & $14.3 \pm 11.8$ & $38 \pm 31.6$ & $80.1 \pm 67.3$ & $y=8.72 x-31.2$ & 0.89 & Increasing \\
\hline Hex-Cer_(37:1) & $0.0494 \pm 0.148$ & $0.167 \pm 0.252$ & $0.0497 \pm 0.141$ & $\mathrm{ND} \pm \mathrm{ND}$ & $\mathrm{y}=0.0000556 \mathrm{x}+0.0884$ & 0.00 & \\
\hline Hex-Cer_(37:2) & $2.12 \pm 2.98$ & $3.57 \pm 5.06$ & $0.717 \pm 2.03$ & $0.957 \pm 2.02$ & $y=-0.235 x+3.64$ & 0.39 & \\
\hline Hex-Cer_(38:0-OH) & $\mathrm{ND} \pm \mathrm{ND}$ & $3.91 \pm 5.51$ & $2.8 \pm 4.29$ & $7.46 \pm 8.35$ & $y=0.657 x-1.19$ & 0.53 & \\
\hline Hex-Cer_(38:1) & $14.8 \pm 16$ & $13.4 \pm 12.3$ & $22.6 \pm 25.1$ & $32.2 \pm 25.2$ & $y=2.27 x+3.35$ & 0.84 & \\
\hline Hex-Cer_(38:2) & $0.569 \pm 0.899$ & $2.09 \pm 1.23$ & $1.81 \pm 1.58$ & $0.813 \pm 0.906$ & $y=0.0167 x+1.19$ & 0.01 & \\
\hline Hex-Cer_(39:0-OH) & $5.62 \pm 3.39$ & $2.48 \pm 3.2$ & $4.86 \pm 13.8$ & $19.3 \pm 21.8$ & $y=1.61 x-4.24$ & 0.54 & \\
\hline Hex-Cer_(39:2) & $20.7 \pm 4.71$ & $21.6 \pm 7.5$ & $24.1 \pm 7.77$ & $32.5 \pm 7.01$ & $y=1.4 x+14$ & 0.83 & Increasing \\
\hline Hex-Cer_(40:0) & $10.1 \pm 12.2$ & $9.47 \pm 10$ & $15.5 \pm 15$ & $19.7 \pm 9.5$ & $y=1.29 x+3.82$ & 0.87 & \\
\hline Hex-Cer_(40:0-OH) & $\mathrm{ND} \pm \mathrm{ND}$ & $5 \pm 15$ & $\mathrm{ND} \pm \mathrm{ND}$ & $\mathrm{ND} \pm \mathrm{ND}$ & & & \\
\hline Hex-Cer_(40:1) & $174 \pm 204$ & $221 \pm 120$ & $275 \pm 249$ & $403 \pm 206$ & $y=27.4 x+58.3 *$ & 0.94 & Increasing \\
\hline Hex-Cer_(40:1-OH) & $7.04 \pm 2.48$ & $6.38 \pm 2.94$ & $7.95 \pm 2.57$ & $5.14 \pm 1.51$ & $y=-0.153 x+7.8$ & 0.20 & \\
\hline Hex-Cer_(40:2) & $0.266 \pm 0.178$ & $0.854 \pm 0.804$ & $0.806 \pm 1.66$ & $0.672 \pm 1.21$ & $y=0.0433 x+0.318$ & 0.32 & \\
\hline Hex-Cer_(40:2-OH) & $2.1 \pm 1.32$ & $2.17 \pm 0.891$ & $2.76 \pm 1.9$ & $1.86 \pm 1.91$ & $y=-0.00481 x+2.26$ & 0.00 & \\
\hline Hex-Cer_(41:0-OH) & $23.9 \pm 8.7$ & $33 \pm 10.5$ & $26.8 \pm 4.88$ & $20.1 \pm 7.48$ & $y=-0.652 x+30.9$ & 0.17 & \\
\hline Hex-Cer $(41: 1)$ & $4.01 \pm 1.31$ & $5.78 \pm 1.62$ & $7.62 \pm 3.75$ & $17.7 \pm 5.68$ & $y=1.59 x-3.38$ & 0.82 & Increasing \\
\hline Hex-Cer_(41:2) & $4.6 \pm 1.03$ & $4.78 \pm 1.62$ & $3.34 \pm 1.5$ & $3.71 \pm 1.89$ & $y=-0.152 x+5.27$ & 0.59 & \\
\hline Hex-Cer_(42:0) & $0.394 \pm 0.271$ & $0.329 \pm 0.289$ & $0.466 \pm 0.227$ & $0.705 \pm 0.432$ & $y=0.0396 x+0.17$ & 0.71 & \\
\hline Hex-Cer_(42:0-OH) & $21.8 \pm 14.5$ & $21.3 \pm 6.34$ & $30.3 \pm 20.7$ & $34.6 \pm 14.3$ & $y=1.76 x+13.6$ & 0.88 & \\
\hline Hex-Cer_(42:1) & $14.1 \pm 4.31$ & $20.5 \pm 5.26$ & $22.5 \pm 10.5$ & $34.5 \pm 12.3$ & $y=2.34 x+4.99^{*}$ & 0.92 & Increasing \\
\hline Hex-Cer_(42:2) & $7.61 \pm 2.29$ & $11.3 \pm 3.91$ & $14.3 \pm 7.05$ & $23.1 \pm 7.39$ & $y=1.83 x+0.061 *$ & 0.93 & Increasing \\
\hline Hex-Cer_(42:2-OH) & $4.99 \pm 1.66$ & $5.86 \pm 2.35$ & $5.19 \pm 1.86$ & $7.37 \pm 3.43$ & $y=0.24 x+4.02$ & 0.60 & \\
\hline Hex-Cer_(43:0) & $0.0149 \pm 0.0296$ & $0.0155 \pm 0.0308$ & $0.145 \pm 0.175$ & $0.129 \pm 0.153$ & $\mathrm{y}=0.0175 \mathrm{x}-0.0576$ & 0.74 & \\
\hline Hex-Cer $(43: 0-\mathrm{OH})$ & $0.416 \pm 0.255$ & $0.583 \pm 0.36$ & $0.338 \pm 0.18$ & $0.32 \pm 0.277$ & $y=-0.0197 x+0.565$ & 0.33 & \\
\hline Hex-Cer_(43:1) & $1.69 \pm 0.642$ & $3.13 \pm 1.14$ & $3.47 \pm 1.46$ & $7.28 \pm 2.91$ & $y=0.634 x-0.955$ & 0.86 & Increasing \\
\hline Hex-Cer_(43:2) & $0.866 \pm 0.212$ & $0.858 \pm 0.321$ & $0.799 \pm 0.314$ & $1.24 \pm 0.718$ & $y=0.0394 x+0.64$ & 0.46 & \\
\hline LPC_(14:0) & $1.4 \pm 0.645$ & $1.58 \pm 0.732$ & $2.09 \pm 0.96$ & $2.96 \pm 1.34$ & $y=0.192 x+0.537 *$ & 0.92 & Increasing \\
\hline LPC_(15:0) & $6.02 \pm 1.49$ & $7.53 \pm 2.48$ & $7.69 \pm 2.62$ & $8.38 \pm 2.62$ & $y=0.268 x+5.35$ & 0.88 & Increasing \\
\hline LPC_(16:0) & $1430 \pm 246$ & $1560 \pm 272$ & $1690 \pm 300$ & $1900 \pm 427$ & $y=57 x+1210^{*}$ & 0.98 & Increasing \\
\hline LPC_(16:1) & $8.56 \pm 4.67$ & $11.3 \pm 6.3$ & $19.6 \pm 8.38$ & $33.1 \pm 10.5$ & $y=3.03 x-5.07 *$ & 0.92 & Increasing \\
\hline LPC_(17:0) & $64.5 \pm 10.8$ & $72.2 \pm 13.1$ & $74.9 \pm 11.2$ & $75.6 \pm 27.5$ & $y=1.33 x+61.6$ & 0.84 & Increasing \\
\hline LPC_(17:1) & $0.395 \pm 0.314$ & $0.576 \pm 0.537$ & $2.2 \pm 1.09$ & $6.08 \pm 1.62$ & $y=0.692 x-2.98$ & 0.83 & Increasing \\
\hline LPC_(18:0) & $3720 \pm 504$ & $3550 \pm 634$ & $3450 \pm 619$ & $3300 \pm 520$ & $y=-50.4 x+3890 *$ & 0.99 & Decreasing \\
\hline LPC_(18:1) & $252 \pm 47.9$ & $247 \pm 56.4$ & $332 \pm 51.2$ & $549 \pm 121$ & $y=36.1 x+68.5$ & 0.79 & \\
\hline LPC (18:2) & $266 \pm 52.6$ & $232 \pm 46.8$ & $217 \pm 19.7$ & $198 \pm 38.2$ & $y=-8.11 x+290 *$ & 0.97 & Decreasing \\
\hline LPC_(18:3) & $80 \pm 15.2$ & $88.6 \pm 22.2$ & $93 \pm 19.4$ & $102 \pm 22.5$ & $y=2.61 x+71 *$ & 0.98 & Increasing \\
\hline LPC $(18: 4)$ & $0.515 \pm 0.37$ & $0.758 \pm 0.481$ & $1.45 \pm 0.744$ & $3.08 \pm 1.42$ & $\mathrm{y}=0.311 \mathrm{x}-0.926$ & 0.88 & Increasing \\
\hline
\end{tabular}


Table 1. Cont

\begin{tabular}{|c|c|c|c|c|c|c|c|}
\hline & Diet 1 & Diet 2 & Diet 3 & Diet 4 & Trendline Equation & $\mathbf{R}^{2}$ & $\begin{array}{l}\text { Successive Change } \\
\text { Across Groups }\end{array}$ \\
\hline LPC_(19:0) & $47.8 \pm 5.54$ & $37.7 \pm 12.1$ & $36.2 \pm 8.19$ & $40.1 \pm 12.9$ & $y=-0.911 x+47.4$ & 0.38 & \\
\hline LPC_(20:0) & $45.5 \pm 12$ & $29 \pm 9.84$ & $22.8 \pm 4.24$ & $18.5 \pm 5.39$ & $y=-3.23 x+53.7 *$ & 0.90 & Decreasing \\
\hline LPC_(20:4) & $344 \pm 121$ & $289 \pm 32.6$ & $288 \pm 82.8$ & $282 \pm 53.7$ & $y=-6.93 x+354$ & 0.69 & Decreasing \\
\hline LPC_(20:5) & $26.9 \pm 9.49$ & $21.5 \pm 4.39$ & $20.2 \pm 2.89$ & $21 \pm 5.62$ & $y=-0.704 x+27.8$ & 0.65 & \\
\hline LPC_(21:0) & $1.11 \pm 0.227$ & $0.89 \pm 0.305$ & $0.75 \pm 0.257$ & $0.896 \pm 0.324$ & $\mathrm{y}=-0.029 \mathrm{x}+1.13$ & 0.46 & \\
\hline LPC_(22:4) & $5.12 \pm 2.83$ & $3.37 \pm 0.794$ & $3.21 \pm 0.909$ & $3.52 \pm 1$ & $y=-0.184 x+5.21$ & 0.52 & \\
\hline LPC_(22:5) & $12.3 \pm 9.44$ & $6.01 \pm 2.43$ & $5.97 \pm 2.51$ & $9.46 \pm 4.97$ & $y=-0.317 x+10.9$ & 0.13 & \\
\hline LPC_(22:6) & $21.1 \pm 13$ & $14.4 \pm 3.33$ & $15.4 \pm 4.74$ & $26.5 \pm 5.75$ & $\mathrm{y}=0.637 \mathrm{x}+14.5$ & 0.16 & \\
\hline LPE_(16:0) & $9.94 \pm 2.69$ & $11.4 \pm 3.82$ & $16.7 \pm 4.93$ & $25.8 \pm 11.7$ & $y=1.96 x+0.977^{*}$ & 0.91 & Increasing \\
\hline LPE $(16: 1)$ & $\mathrm{ND} \pm \mathrm{ND}$ & $0.0351 \pm 0.0709$ & $0.0116 \pm 0.0329$ & $0.177 \pm 0.163$ & $y=0.0263 x-0.162$ & 0.63 & \\
\hline LPE_(17:0) & $0.637 \pm 0.392$ & $0.724 \pm 0.401$ & $1.1 \pm 0.151$ & $1.61 \pm 0.982$ & $y=0.122 x+0.0842 *$ & 0.92 & Increasing \\
\hline LPE_(18:0) & $50.7 \pm 12.9$ & $50.3 \pm 14.6$ & $57.1 \pm 12.6$ & $67.7 \pm 29.3$ & $y=2.14 x+40.1$ & 0.84 & \\
\hline LPE_(18:1) & $5.94 \pm 1.16$ & $4.7 \pm 1.21$ & $6.6 \pm 1.36$ & $11 \pm 3.41$ & $y=0.633 x+2.22$ & 0.65 & \\
\hline LPE_(18:2) & $5.04 \pm 3.8$ & $2.76 \pm 1.33$ & $2.93 \pm 1.15$ & $2.42 \pm 1.28$ & $y=-0.285 x+5.47$ & 0.70 & \\
\hline LPE (18:3) & $1.85 \pm 0.528$ & $2.14 \pm 0.76$ & $2.96 \pm 0.944$ & $4.22 \pm 1.93$ & $y=0.294 x+0.546 *$ & 0.93 & Increasing \\
\hline LPE_(20:0) & $0.444 \pm 0.114$ & $0.358 \pm 0.169$ & $0.31 \pm 0.121$ & $0.247 \pm 0.17$ & $y=-0.0237 x+0.521 *$ & 0.99 & Decreasing \\
\hline LPE_(20:3) & $0.472 \pm 0.645$ & $0.556 \pm 0.49$ & $0.945 \pm 0.831$ & $1.49 \pm 1.22$ & $y=0.128 x-0.11^{*}$ & 0.92 & Increasing \\
\hline LPE $(20: 4)$ & $22.9 \pm 12.3$ & $17.9 \pm 4.53$ & $20.8 \pm 7.66$ & $28.6 \pm 11.8$ & $y=0.741 x+16.9$ & 0.33 & \\
\hline LPE_(20:5) & $1.53 \pm 1.12$ & $0.932 \pm 0.388$ & $0.827 \pm 0.252$ & $0.645 \pm 0.248$ & $y=-0.102 x+1.77$ & 0.86 & Decreasing \\
\hline LPE_(22:4) & $0.264 \pm 0.791$ & $\mathrm{ND} \pm \mathrm{ND}$ & $0.0209 \pm 0.0389$ & $0.35 \pm 0.382$ & $y=0.00267 x+0.19$ & 0.00 & \\
\hline LPI_(16:0) & $64.1 \pm 21.4$ & $82.6 \pm 36.1$ & $113 \pm 39.7$ & $103 \pm 45$ & $y=5.45 x+49$ & 0.76 & \\
\hline LPI_(17:0) & $5.04 \pm 3.17$ & $6.56 \pm 3.13$ & $7.63 \pm 3.8$ & $5.1 \pm 4.85$ & $y=0.0463 x+5.73$ & 0.02 & \\
\hline LPI_(18:0) & $1210 \pm 404$ & $1510 \pm 647$ & $1510 \pm 376$ & $1200 \pm 547$ & $y=-1.11 x+1370$ & 0.00 & \\
\hline LPI_(18:1) & $46.2 \pm 22.3$ & $47.6 \pm 17.7$ & $57.4 \pm 21.6$ & $59.5 \pm 26.8$ & $y=1.84 x+38.6 *$ & 0.90 & Increasing \\
\hline LPI_(18:2) & $18.9 \pm 10.4$ & $17.8 \pm 8$ & $16.9 \pm 5.86$ & $7.43 \pm 4.45$ & $y=-1.31 x+25.3$ & 0.74 & Decreasing \\
\hline LPI $(20: 0)$ & $0.0835 \pm 0.1$ & $\mathrm{ND} \pm \mathrm{ND}$ & $0.18 \pm 0.264$ & $0.0895 \pm 0.127$ & $y=0.00319 x+0.0918$ & 0.06 & \\
\hline LPI_(20:2) & $1.7 \pm 1.09$ & $1.18 \pm 1.36$ & $1.09 \pm 0.93$ & $0.661 \pm 1.15$ & $y=-0.119 x+2.07 *$ & 0.94 & Decreasing \\
\hline LPI_(20:3) & $25.8 \pm 14.7$ & $47.1 \pm 27.2$ & $85.6 \pm 35.3$ & $125 \pm 49.4$ & $y=12.4 x-24.4 *$ & 0.98 & Increasing \\
\hline LPI_(20:4) & $288 \pm 102$ & $453 \pm 238$ & $447 \pm 108$ & $360 \pm 145$ & $y=7.78 x+328$ & 0.12 & \\
\hline LPI_(22:4) & $4.72 \pm 2.12$ & $3.96 \pm 2.88$ & $4.26 \pm 2.1$ & $3.26 \pm 1.91$ & $y=-0.151 x+5.21$ & 0.74 & \\
\hline LPI (22:5) & $1.15 \pm 1.67$ & $1.51 \pm 1.76$ & $0.418 \pm 0.701$ & $1.98 \pm 1.41$ & $\mathrm{y}=0.0518 \mathrm{x}+0.868$ & 0.08 & \\
\hline LPI_(22:6) & $2.06 \pm 1.55$ & $2.04 \pm 1.58$ & $0.605 \pm 0.968$ & $2.65 \pm 2.37$ & $y=0.0124 x+1.74$ & 0.00 & \\
\hline Lyso_CL_(52:01) & $0.698 \pm 1.02$ & $1.75 \pm 1.42$ & $1.51 \pm 0.755$ & $1.99 \pm 1.82$ & $y=0.135 x+0.457$ & 0.70 & \\
\hline Lyso_CL_(52:02) & $11.4 \pm 10.4$ & $13.7 \pm 7.67$ & $10.7 \pm 4.9$ & $12.5 \pm 11.6$ & $y=0.0111 x+12$ & 0.00 & \\
\hline Lyso_CL_(52:03) & $50.5 \pm 42.8$ & $32.9 \pm 12.7$ & $18.7 \pm 8.32$ & $17.2 \pm 14.1$ & $y=-4.23 x+62.2 *$ & 0.90 & Decreasing \\
\hline Lyso_CL_(52:04) & $30.3 \pm 36.6$ & $19.7 \pm 9.87$ & $9.23 \pm 4.21$ & $10.4 \pm 9.01$ & $y=-2.6 x+37.3$ & 0.86 & \\
\hline Lyso CL (52:05) & $\mathrm{ND} \pm \mathrm{ND}$ & $\mathrm{ND} \pm \mathrm{ND}$ & $\mathrm{ND} \pm \mathrm{ND}$ & $0.785 \pm 1.84$ & & & \\
\hline Lyso_CL_(53:04) & $\mathrm{ND} \pm \mathrm{ND}$ & $0.154 \pm 0.261$ & $0.221 \pm 0.404$ & $1.55 \pm 1.72$ & $y=0.259 x-1.69$ & 0.79 & \\
\hline
\end{tabular}


Table 1. Cont

\begin{tabular}{|c|c|c|c|c|c|c|c|}
\hline & Diet 1 & Diet 2 & Diet 3 & Diet 4 & Trendline Equation & $\mathbf{R}^{2}$ & $\begin{array}{l}\text { Successive Change } \\
\text { Across Groups }\end{array}$ \\
\hline Lyso_CL_(54:02) & $\mathrm{ND} \pm \mathrm{ND}$ & $0.0851 \pm 0.178$ & $0.0412 \pm 0.117$ & $0.655 \pm 1.15$ & $y=0.106 x-0.689$ & 0.69 & \\
\hline Lyso CL (54:03) & $24.8 \pm 26.6$ & $13.2 \pm 7.1$ & $9.89 \pm 3.88$ & $19.5 \pm 18.6$ & $\mathrm{y}=-0.711 \mathrm{x}+22.3$ & 0.14 & \\
\hline Lyso_CL_(54:04) & $172 \pm 166$ & $94.9 \pm 32.4$ & $67.3 \pm 25.2$ & $87 \pm 72.4$ & $y=-10.5 x+185$ & 0.63 & \\
\hline Lyso_CL_(54:05) & $405 \pm 411$ & $234 \pm 94$ & $144 \pm 60.4$ & $133 \pm 104$ & $y=-33.6 x+486$ & 0.87 & Decreasing \\
\hline Lyso_CL_(54:06) & $548 \pm 555$ & $247 \pm 117$ & $122 \pm 47.8$ & $65.7 \pm 51.8$ & $y=-58.2 x+691$ & 0.89 & Decreasing \\
\hline Lyso_CL_(56:05) & $42.9 \pm 55.3$ & $13.1 \pm 3.27$ & $4.18 \pm 2.41$ & $13.5 \pm 16.1$ & $y=-3.6 x+45.9$ & 0.55 & \\
\hline Lyso_CL_(56:06) & $135 \pm 153$ & $42.7 \pm 13.4$ & $20.9 \pm 8.33$ & $40.2 \pm 42.2$ & $y=-11.3 x+146$ & 0.60 & \\
\hline PA_(30:0) & $1.19 \pm 2.41$ & $2.63 \pm 2.77$ & $6.15 \pm 4.83$ & $30 \pm 23.7$ & $y=3.33 x-15.5$ & 0.74 & Increasing \\
\hline PA_(30:1) & $17.2 \pm 17.8$ & $17.1 \pm 11.8$ & $20.7 \pm 9.98$ & $73.9 \pm 41.3$ & $y=6.43 x-17$ & 0.65 & \\
\hline PA_(32:1) & $43.2 \pm 46.5$ & $56.3 \pm 17.7$ & $84.6 \pm 30.3$ & $291 \pm 143$ & $y=28.6 x-99.9$ & 0.74 & Increasing \\
\hline PA_(32:2) & $223 \pm 216$ & $180 \pm 96$ & $154 \pm 86.4$ & $166 \pm 70.4$ & $y=-7.3 x+237$ & 0.71 & \\
\hline PA_(34:1) & $387 \pm 401$ & $402 \pm 135$ & $538 \pm 176$ & $1010 \pm 307$ & $y=74.3 x+16.2$ & 0.79 & Increasing \\
\hline PA_(34:2) & $2330 \pm 2170$ & $1990 \pm 756$ & $1600 \pm 787$ & $1230 \pm 358$ & $y=-137 x+2830 *$ & 1.00 & Decreasing \\
\hline PA_(36:1) & $1440 \pm 924$ & $1640 \pm 435$ & $1410 \pm 877$ & $1240 \pm 323$ & $y=-30.7 x+1670$ & 0.43 & \\
\hline PA_(36:2) & $3310 \pm 1750$ & $2480 \pm 918$ & $2250 \pm 1360$ & $1910 \pm 1020$ & $y=-164 x+3740 *$ & 0.92 & Decreasing \\
\hline PA_(36:4) & $10,300 \pm 9970$ & $6760 \pm 2840$ & $7130 \pm 3790$ & $9580 \pm 4540$ & $y=-66.3 x+8950$ & 0.02 & \\
\hline PA_(38:3) & $1390 \pm 910$ & $1150 \pm 544$ & $1590 \pm 683$ & $2590 \pm 1230$ & $y=150 x+535$ & 0.68 & \\
\hline PA $(38: 4)$ & $42,400 \pm 40,100$ & $27,100 \pm 11,700$ & $25,400 \pm 17,000$ & $23,000 \pm 7630$ & $\mathrm{y}=-2220 \mathrm{x}+46,400$ & 0.78 & Decreasing \\
\hline PA_(38:5) & $3280 \pm 2800$ & $2230 \pm 911$ & $2430 \pm 1180$ & $3310 \pm 1300$ & $y=10.7 x+2730$ & 0.00 & \\
\hline PA_(40:5) & $1730 \pm 1530$ & $838 \pm 336$ & $871 \pm 449$ & $1110 \pm 412$ & $y=-67.7 x+1650$ & 0.33 & \\
\hline PC_(30:0) & $6.27 \pm 6.94$ & $21.5 \pm 23.9$ & $39.3 \pm 18.4$ & $81.4 \pm 23.1$ & $y=9.01 x-31.8^{*}$ & 0.94 & Increasing \\
\hline PC_(30:1) & $8.63 \pm 6.24$ & $12.9 \pm 8.52$ & $40.6 \pm 32.6$ & $188 \pm 120$ & $y=21 x-97.8$ & 0.74 & Increasing \\
\hline PC $(31: 0)$ & $54.7 \pm 17.4$ & $78.7 \pm 33.5$ & $85.4 \pm 22.6$ & $179 \pm 44.5$ & $\mathrm{y}=14.1 \mathrm{x}-8.1$ & 0.80 & Increasing \\
\hline PC_(32:0) & $1750 \pm 251$ & $1930 \pm 493$ & $1650 \pm 237$ & $1950 \pm 234$ & $y=11.9 x+1730$ & 0.08 & \\
\hline PC_(32:1) & $222 \pm 110$ & $352 \pm 101$ & $1240 \pm 835$ & $4860 \pm 1550$ & $y=548 x-2530$ & 0.77 & Increasing \\
\hline PC $(32: 2)$ & $565 \pm 249$ & $496 \pm 222$ & $646 \pm 223$ & $1350 \pm 659$ & $y=92.8 x+54.5$ & 0.67 & \\
\hline PC_(33:0) & $28.2 \pm 8.55$ & $39.9 \pm 13$ & $49.6 \pm 7.5$ & $102 \pm 25.7$ & $y=8.56 x-10.6$ & 0.84 & Increasing \\
\hline PC_(33:1) & $148 \pm 45.4$ & $267 \pm 71.4$ & $540 \pm 136$ & $1700 \pm 388$ & $y=183 x-733$ & 0.80 & Increasing \\
\hline PC_(33:2) & $470 \pm 56.2$ & $515 \pm 187$ & $574 \pm 241$ & $566 \pm 180$ & $y=12.9 x+433$ & 0.85 & \\
\hline PC_(34:0) & $789 \pm 267$ & $732 \pm 165$ & $615 \pm 45.6$ & $644 \pm 83.3$ & $y=-20.4 x+851$ & 0.79 & \\
\hline PC_(34:1) & $4160 \pm 763$ & $5580 \pm 931$ & $8080 \pm 829$ & $15,100 \pm 1050$ & $y=1310 x-1780$ & 0.88 & Increasing \\
\hline PC_(34:2) & $11,900 \pm 2050$ & $14,700 \pm 1880$ & $15,200 \pm 1930$ & $20,400 \pm 2030$ & $y=963 x+8180$ & 0.90 & Increasing \\
\hline PC_(34:3) & $3270 \pm 1090$ & $3620 \pm 834$ & $5400 \pm 998$ & $9050 \pm 1860$ & $y=708 x-82.3$ & 0.87 & Increasing \\
\hline PC $(35: 0)$ & $28.3 \pm 9.27$ & $32.3 \pm 9.11$ & $38.3 \pm 6.8$ & $71.2 \pm 15.2$ & $\mathrm{y}=4.99 \mathrm{x}+4.36$ & 0.79 & Increasing \\
\hline PC_(35:1) & $108 \pm 32$ & $166 \pm 36.7$ & $297 \pm 52.6$ & $891 \pm 153$ & $y=91.9 x-337$ & 0.79 & Increasing \\
\hline PC_(35:2) & $1240 \pm 238$ & $1530 \pm 305$ & $1530 \pm 433$ & $1640 \pm 369$ & $y=44.4 x+1150$ & 0.82 & \\
\hline PC $(36: 0)$ & $76.2 \pm 27.6$ & $66.4 \pm 16.6$ & $55.2 \pm 22.9$ & $83.4 \pm 16.5$ & $y=0.385 x+67.4$ & 0.01 & \\
\hline PC_(36:1) & $3060 \pm 753$ & $3610 \pm 756$ & $5450 \pm 944$ & $11,600 \pm 974$ & $y=1020 x-1850$ & 0.82 & Increasing \\
\hline
\end{tabular}


Table 1. Cont

\begin{tabular}{|c|c|c|c|c|c|c|c|}
\hline & Diet 1 & Diet 2 & Diet 3 & Diet 4 & Trendline Equation & $\mathbf{R}^{2}$ & $\begin{array}{l}\text { Successive Change } \\
\text { Across Groups }\end{array}$ \\
\hline PC_(36:2) & $6650 \pm 1180$ & $7290 \pm 1320$ & $7890 \pm 1490$ & $10,700 \pm 954$ & $y=472 x+4520$ & 0.85 & Increasing \\
\hline PC_(36:3) & $8480 \pm 912$ & $9060 \pm 1570$ & $11,800 \pm 1920$ & $23,900 \pm 2150$ & $y=1810 x-573$ & 0.77 & Increasing \\
\hline PC_( $36: 4)$ & $18,900 \pm 3120$ & $20,200 \pm 2700$ & $19,500 \pm 1190$ & $27,500 \pm 1900$ & $y=930 x+14,400$ & 0.65 & \\
\hline PC_( $37: 0)$ & $0.557 \pm 0.4$ & $0.542 \pm 0.232$ & $0.338 \pm 0.231$ & $0.689 \pm 0.551$ & $y=0.00711 x+0.477$ & 0.03 & \\
\hline PC_(37:1) & $40.9 \pm 20.8$ & $38 \pm 19.4$ & $75.1 \pm 38.1$ & $283 \pm 96.2$ & $y=28.3 x-107$ & 0.71 & \\
\hline PC_(37:2) & $301 \pm 88.2$ & $217 \pm 62.9$ & $210 \pm 61.6$ & $301 \pm 56.6$ & $y=-0.259 x+259$ & 0.00 & \\
\hline PC_(37:3) & $83.4 \pm 17.3$ & $86.2 \pm 25.1$ & $131 \pm 33.9$ & $160 \pm 31.9$ & $y=10.2 x+37.3^{*}$ & 0.92 & Increasing \\
\hline PC_ $(37: 4)$ & $2810 \pm 648$ & $3180 \pm 738$ & $2520 \pm 674$ & $2430 \pm 461$ & $y=-66.7 x+3250$ & 0.47 & \\
\hline PC_(37:5) & $157 \pm 59.9$ & $171 \pm 36.4$ & $270 \pm 103$ & $445 \pm 77.3$ & $y=35.7 x-12.1$ & 0.88 & Increasing \\
\hline PC_(37:6) & $85 \pm 34.3$ & $76 \pm 37.5$ & $55.8 \pm 29.9$ & $101 \pm 29.7$ & $y=1.03 x+71.6$ & 0.04 & \\
\hline PC_( $38: 0)$ & $1.83 \pm 0.323$ & $1.77 \pm 0.432$ & $1.79 \pm 0.773$ & $2.67 \pm 0.91$ & $y=0.0941 x+1.3$ & 0.56 & \\
\hline PC_(38:1) & $39.4 \pm 20.9$ & $14.1 \pm 12.8$ & $28.9 \pm 21.3$ & $103 \pm 42.3$ & $y=7.61 x-11.9$ & 0.46 & \\
\hline PC_(38:2) & $649 \pm 137$ & $521 \pm 186$ & $493 \pm 137$ & $871 \pm 171$ & $y=23.6 x+453$ & 0.23 & \\
\hline PC_(38:3) & $3030 \pm 346$ & $3260 \pm 708$ & $5020 \pm 1050$ & $12,200 \pm 1770$ & $y=1080 x-2420$ & 0.77 & Increasing \\
\hline PC_(38:4) & $10,300 \pm 2010$ & $11,700 \pm 2240$ & $10,200 \pm 1090$ & $12,100 \pm 2290$ & $y=144 x+9970$ & 0.27 & \\
\hline PC_(38:5) & $6010 \pm 2050$ & $5500 \pm 1900$ & $5290 \pm 916$ & $8730 \pm 1930$ & $y=294 x+4130$ & 0.41 & \\
\hline PC_(38:6) & $16,000 \pm 3530$ & $14,000 \pm 1550$ & $12,900 \pm 1690$ & $18,900 \pm 2600$ & $y=281 x+13,300$ & 0.14 & \\
\hline PC_(40:2) & $19.1 \pm 8.48$ & $14.8 \pm 4.37$ & $13.2 \pm 3.21$ & $20.1 \pm 5.31$ & $y=0.0519 x+16.4$ & 0.00 & \\
\hline PC_(40:3) & $52.8 \pm 15$ & $44 \pm 15.1$ & $52.7 \pm 13.6$ & $124 \pm 22.6$ & $y=8.23 x+5.39$ & 0.59 & \\
\hline PC_(40:4) & $1200 \pm 402$ & $884 \pm 230$ & $726 \pm 182$ & $665 \pm 175$ & $y=-65.3 x+1370 *$ & 0.90 & Decreasing \\
\hline PC_(40:5) & $947 \pm 577$ & $704 \pm 574$ & $514 \pm 208$ & $980 \pm 459$ & $y=-3.37 x+812$ & 0.00 & \\
\hline PC_(40:6) & $3800 \pm 936$ & $3630 \pm 913$ & $3090 \pm 720$ & $4370 \pm 960$ & $y=43.3 x+3390$ & 0.08 & \\
\hline PC_C18(plas)-18:1 & $\mathrm{ND} \pm \mathrm{ND}$ & $\mathrm{ND} \pm \mathrm{ND}$ & $\mathrm{ND} \pm \mathrm{ND}$ & $0.766 \pm 1.43$ & & & \\
\hline PE_(30:0) & $4.78 \pm 10.7$ & $2.19 \pm 4.47$ & $\mathrm{ND} \pm \mathrm{ND}$ & $1.82 \pm 5.14$ & $y=-0.323 x+5.26$ & 0.68 & \\
\hline PE_(32:1) & $12.4 \pm 20.9$ & $26 \pm 38.5$ & $468 \pm 553$ & $1920 \pm 896$ & $y=228 x-1140$ & 0.78 & Increasing \\
\hline PE_(34:0) & $80.3 \pm 34.4$ & $106 \pm 24$ & $54.8 \pm 20.8$ & $30.5 \pm 35.6$ & $y=-7.43 x+125$ & 0.63 & \\
\hline PE_(34:1) & $4110 \pm 2220$ & $4930 \pm 1800$ & $9030 \pm 1940$ & $18,100 \pm 6000$ & $y=1710 x-4010$ & 0.86 & Increasing \\
\hline PE_(34:2) & $71,600 \pm 27,000$ & $69,300 \pm 42,400$ & $48,600 \pm 14,200$ & $29,500 \pm 12,000$ & $y=-5440 x+96,400 *$ & 0.92 & Decreasing \\
\hline PE_(35:1) & $4.9 \pm 2$ & $10.6 \pm 3.84$ & $23.9 \pm 7.89$ & $42.4 \pm 13$ & $y=4.66 x-15.2 *$ & 0.95 & Increasing \\
\hline PE_(36:0) & $24.4 \pm 11.3$ & $35 \pm 15$ & $22.8 \pm 6.68$ & $17 \pm 12.8$ & $y=-1.27 x+34.5$ & 0.35 & \\
\hline PE_(36:1) & $6560 \pm 4060$ & $5730 \pm 2600$ & $6590 \pm 1730$ & $11,300 \pm 4870$ & $y=559 x+3270$ & 0.59 & \\
\hline PE_(36:2) & $46,300 \pm 18,900$ & $36,900 \pm 20,900$ & $24,400 \pm 6230$ & $19,300 \pm 7930$ & $y=-3460 x+58,200 *$ & 0.98 & Decreasing \\
\hline PE_(36:3) & $39,000 \pm 15,000$ & $28,800 \pm 17,100$ & $20,000 \pm 4520$ & $14,300 \pm 5490$ & $y=-3070 x+49,000 *$ & 0.99 & Decreasing \\
\hline PE_(36:4) & $110,000 \pm 44,700$ & $107,000 \pm 49,200$ & $96,600 \pm 12,500$ & $126,000 \pm 34,500$ & $y=1390 x+99,200$ & 0.16 & \\
\hline PE_(38:1) & $219 \pm 130$ & $144 \pm 84.5$ & $115 \pm 22$ & $121 \pm 46.7$ & $y=-12 x+241$ & 0.76 & \\
\hline PE_(38:2) & $1530 \pm 645$ & $822 \pm 467$ & $539 \pm 98.4$ & $535 \pm 140$ & $y=-121 x+1780$ & 0.81 & Decreasing \\
\hline PE (38:3) & $13400 \pm 4930$ & $13,000 \pm 7700$ & $14,400 \pm 2710$ & $17,700 \pm 3860$ & $y=530 x+10,600$ & 0.75 & \\
\hline PE_(38:4) & $165,000 \pm 74,800$ & $148,000 \pm 59,300$ & $113,000 \pm 20,200$ & $118,000 \pm 26,000$ & $y=-6520 x+186,000$ & 0.84 & \\
\hline
\end{tabular}


Table 1. Cont

\begin{tabular}{|c|c|c|c|c|c|c|c|}
\hline & Diet 1 & Diet 2 & Diet 3 & Diet 4 & Trendline Equation & $\mathbf{R}^{2}$ & $\begin{array}{l}\text { Successive Change } \\
\text { Across Groups }\end{array}$ \\
\hline PE_(38:5) & $72,900 \pm 38,400$ & $52,700 \pm 23,200$ & $39,700 \pm 7150$ & $44,700 \pm 11,600$ & $y=-3610 x+80,200$ & 0.74 & \\
\hline PE_(38:6) & $25,000 \pm 12,000$ & $18,200 \pm 8270$ & $12,300 \pm 2890$ & $19,100 \pm 5400$ & $y=-874 x+25,300$ & 0.34 & \\
\hline PG_(32:0) & $1.64 \pm 0.748$ & $1.59 \pm 0.834$ & $5.29 \pm 4.05$ & $11 \pm 3.46$ & $\mathrm{y}=1.18 \mathrm{x}-4.12$ & 0.86 & \\
\hline PG_(33:0) & $\mathrm{ND} \pm \mathrm{ND}$ & $\mathrm{ND} \pm \mathrm{ND}$ & $\mathrm{ND} \pm \mathrm{ND}$ & $0.0213 \pm 0.0434$ & & & \\
\hline PG_(34:1) & $\mathrm{ND} \pm \mathrm{ND}$ & $\mathrm{ND} \pm \mathrm{ND}$ & $2.24 \pm 3.19$ & $65.1 \pm 51.8$ & $y=23.3 x-207$ & 1.00 & \\
\hline PG_(35:1) & $0.256 \pm 0.325$ & $0.467 \pm 0.67$ & $0.816 \pm 0.888$ & $1.62 \pm 0.913$ & $y=0.164 x-0.469 *$ & 0.91 & Increasing \\
\hline PG_(36:0) & $11.8 \pm 3.75$ & $25.6 \pm 18$ & $13.4 \pm 4.49$ & $7.6 \pm 5.1$ & $y=-0.919 x+21.6$ & 0.17 & \\
\hline PG_(36:1) & $0.0408 \pm 0.122$ & $1.84 \pm 2.72$ & $0.525 \pm 0.62$ & $0.294 \pm 0.831$ & $y=-0.0206 x+0.832$ & 0.01 & \\
\hline PG_(36:2) & $125 \pm 25.3$ & $169 \pm 81.3$ & $106 \pm 43.1$ & $53.5 \pm 21$ & $y=-10.3 x+192$ & 0.56 & \\
\hline PG_(36:3) & $48.8 \pm 14.2$ & $62.3 \pm 30$ & $32.3 \pm 9.53$ & $28.1 \pm 20.5$ & $y=-3.41 x+69$ & 0.57 & \\
\hline PG_(36:4) & $25.6 \pm 13.6$ & $45.9 \pm 26.9$ & $33.6 \pm 15.7$ & $29.1 \pm 15.4$ & $y=-0.0667 x+34.1$ & 0.00 & \\
\hline PG_(38:3) & $1.72 \pm 1.42$ & $1.04 \pm 0.753$ & $3.19 \pm 3.43$ & $7.34 \pm 3.64$ & $y=0.704 x-2.06$ & 0.75 & \\
\hline PG_(38:4) & $11.7 \pm 5.76$ & $19.5 \pm 9.9$ & $13.3 \pm 5.22$ & $11.9 \pm 5.31$ & $y=-0.207 x+15.7$ & 0.04 & \\
\hline PG_(38:5) & $6.31 \pm 2.82$ & $9.55 \pm 5.47$ & $6.6 \pm 2.34$ & $7.16 \pm 4.03$ & $y=-0.0148 x+7.52$ & 0.00 & \\
\hline PG_(38:6) & $0.0292 \pm 0.0876$ & $0.0656 \pm 0.0754$ & $0.0529 \pm 0.067$ & $0.0457 \pm 0.0527$ & $y=0.00136 x+0.0379$ & 0.10 & \\
\hline PG_(40:6) & $0.306 \pm 0.378$ & $0.211 \pm 0.434$ & $0.028 \pm 0.0792$ & $0.773 \pm 0.913$ & $y=0.0451 x-0.0156$ & 0.25 & \\
\hline PG_(42:07) & $0.469 \pm 0.411$ & $0.424 \pm 0.714$ & $0.269 \pm 0.332$ & $0.305 \pm 0.316$ & $y=-0.024 x+0.55$ & 0.77 & \\
\hline PG_(42:08) & $0.0147 \pm 0.0192$ & $0.0893 \pm 0.11$ & $0.112 \pm 0.116$ & $\mathrm{ND} \pm \mathrm{ND}$ & $y=0.018 x-0.0415$ & 0.91 & Increasing \\
\hline PG_(42:10) & $0.0638 \pm 0.191$ & $0.314 \pm 0.943$ & $\mathrm{ND} \pm \mathrm{ND}$ & $0.0417 \pm 0.118$ & $y=-0.00954 x+0.209$ & 0.07 & \\
\hline PI_(34:0) & $455 \pm 385$ & $482 \pm 396$ & $354 \pm 237$ & $318 \pm 196$ & $y=-20 x+555$ & 0.78 & \\
\hline PI_(34:1) & $5070 \pm 1420$ & $5070 \pm 909$ & $7620 \pm 1620$ & $10,100 \pm 2670$ & $y=653 x+1970$ & 0.89 & \\
\hline PI_(34:2) & $6510 \pm 3850$ & $9740 \pm 4140$ & $9450 \pm 3880$ & $3260 \pm 1640$ & $y=-372 x+10,100$ & 0.18 & \\
\hline PI_(35:0) & $0.0814 \pm 0.244$ & $0.0917 \pm 0.275$ & $\mathrm{ND} \pm \mathrm{ND}$ & $\mathrm{ND} \pm \mathrm{ND}$ & $y=0.00381 x+0.0677$ & 1.00 & \\
\hline PI_(35:2) & $134 \pm 112$ & $319 \pm 164$ & $261 \pm 137$ & $82.5 \pm 68.9$ & $y=-7.87 x+259$ & 0.06 & \\
\hline PI_(36:1) & $186 \pm 165$ & $450 \pm 292$ & $659 \pm 339$ & $814 \pm 589$ & $y=77.5 x-65.8^{*}$ & 0.99 & Increasing \\
\hline PI_(36:2) & $3680 \pm 2670$ & $5400 \pm 2720$ & $4920 \pm 2300$ & $2020 \pm 1140$ & $y=-202 x+5550$ & 0.22 & \\
\hline PI_(36:4) & $16,000 \pm 6720$ & $25,800 \pm 10,600$ & $27,900 \pm 8740$ & $21,000 \pm 9500$ & $y=633 x+17,800$ & 0.17 & \\
\hline PI_(38:3) & $6340 \pm 4020$ & $10,500 \pm 6710$ & $14,200 \pm 8060$ & $6420 \pm 3950$ & $y=146 x+8250$ & 0.02 & \\
\hline PI_(38:4) & $86,600 \pm 41,000$ & $124,000 \pm 50,900$ & $119,000 \pm 34,000$ & $79,600 \pm 40,400$ & $y=-963 x+110,000$ & 0.02 & \\
\hline PI_(38:5) & $11,100 \pm 5400$ & $15,000 \pm 5750$ & $16,600 \pm 4890$ & $12,400 \pm 5840$ & $y=204 x+12,200$ & 0.08 & \\
\hline PI_(40:3) & $120 \pm 56.6$ & $94 \pm 51.8$ & $49.8 \pm 19$ & $25.2 \pm 8.08$ & $y=-12.2 x+165 *$ & 0.99 & Decreasing \\
\hline PI_(40:4) & $1040 \pm 615$ & $1830 \pm 1050$ & $1380 \pm 608$ & $885 \pm 607$ & $y=-33.9 x+1540$ & 0.08 & \\
\hline PI_(40:6) & $4080 \pm 1020$ & $5270 \pm 1350$ & $4820 \pm 901$ & $6870 \pm 1270$ & $y=293 x+3020$ & 0.75 & \\
\hline PI_(40:8) & $42.9 \pm 25$ & $48.9 \pm 23.4$ & $47.4 \pm 22.1$ & $50.8 \pm 21$ & $y=0.822 x+41.2$ & 0.72 & \\
\hline PS_(32:0) & $0.673 \pm 0.587$ & $0.661 \pm 0.918$ & $0.321 \pm 0.135$ & $0.802 \pm 0.336$ & $y=0.00174 x+0.601$ & 0.00 & \\
\hline PS_(32:1) & $0.00219 \pm 0.00658$ & $0.0339 \pm 0.0329$ & $0.00448 \pm 0.0127$ & $0.00374 \pm 0.0106$ & $y=-0.000917 x+0.0181$ & 0.04 & \\
\hline PS_(33:1) & $1.25 \pm 0.504$ & $1.76 \pm 0.541$ & $1.8 \pm 0.553$ & $1.19 \pm 1.06$ & $y=-0.00519 x+1.54$ & 0.00 & \\
\hline PS_(34:0) & $0.0337 \pm 0.101$ & $0.291 \pm 0.452$ & $0.16 \pm 0.211$ & $0.0675 \pm 0.096$ & $y=-0.0011 x+0.146$ & 0.00 & \\
\hline PS_(34:1) & $\mathrm{ND} \pm \mathrm{ND}$ & $0.0315 \pm 0.0945$ & $\mathrm{ND} \pm \mathrm{ND}$ & $0.73 \pm 0.911$ & $y=0.129 x-0.783$ & 1.00 & \\
\hline PS_(34:2) & $10.3 \pm 4.05$ & $13.8 \pm 4.69$ & $15.3 \pm 4.34$ & $11.7 \pm 5.1$ & $y=0.211 x+11.2$ & 0.11 & \\
\hline PS_(34:3) & $0.051 \pm 0.0821$ & $0.0582 \pm 0.115$ & $0.202 \pm 0.314$ & $0.911 \pm 0.888$ & $y=0.101 x-0.466$ & 0.74 & Increasing \\
\hline PS_(35:2) & $0.215 \pm 0.514$ & $0.379 \pm 0.946$ & $0.0732 \pm 0.207$ & $0.101 \pm 0.286$ & $y=-0.024 x+0.376$ & 0.36 & \\
\hline PS_(36:0) & $0.242 \pm 0.479$ & $0.34 \pm 0.527$ & $0.261 \pm 0.489$ & $0.485 \pm 0.672$ & $y=0.0241 x+0.148$ & 0.58 & \\
\hline PS_(36:1) & $63.9 \pm 28.7$ & $135 \pm 76.6$ & $101 \pm 23.5$ & $75.4 \pm 43$ & $y=0.0185 x+93.7$ & 0.00 & \\
\hline PS_(36:2) & $115 \pm 47$ & $136 \pm 51.5$ & $123 \pm 33.2$ & $83 \pm 23.2$ & $y=-4.04 x+145$ & 0.39 & \\
\hline PS_(38:2) & $0.434 \pm 0.578$ & $1.66 \pm 1.78$ & $0.712 \pm 0.42$ & $0.536 \pm 0.84$ & $y=-0.0238 x+1.02$ & 0.02 & \\
\hline
\end{tabular}


Table 1. Cont.

\begin{tabular}{|c|c|c|c|c|c|c|c|}
\hline & Diet 1 & Diet 2 & Diet 3 & Diet 4 & Trendline Equation & $\mathbf{R}^{2}$ & $\begin{array}{l}\text { Successive Change } \\
\text { Across Groups }\end{array}$ \\
\hline S_(34:1-OH) & $1.47 \pm 0.48$ & $1.72 \pm 0.795$ & $1.08 \pm 0.388$ & $1.35 \pm 0.9$ & $y=-0.037 x+1.69$ & 0.24 & \\
\hline S_(34:2) & $0.592 \pm 0.147$ & $0.769 \pm 0.36$ & $0.402 \pm 0.187$ & $0.327 \pm 0.128$ & $y=-0.043 x+0.852$ & 0.57 & \\
\hline S_(35:0) & $0.0316 \pm 0.0617$ & $0.022 \pm 0.0369$ & $0.0626 \pm 0.123$ & $0.0338 \pm 0.0371$ & $y=0.00175 x+0.0241$ & 0.12 & \\
\hline S_(35:1) & $0.0235 \pm 0.041$ & $0.021 \pm 0.0344$ & $0.0404 \pm 0.0922$ & $0.16 \pm 0.307$ & $y=0.0159 x-0.0603$ & 0.70 & \\
\hline S_(35:1-OH) & $0.209 \pm 0.281$ & $0.127 \pm 0.381$ & $\mathrm{ND} \pm \mathrm{ND}$ & $\mathrm{ND} \pm \mathrm{ND}$ & $y=-0.0304 x+0.318$ & 1.00 & \\
\hline S_(35:2) & $\mathrm{ND} \pm \mathrm{ND}$ & $\mathrm{ND} \pm \mathrm{ND}$ & $0.00482 \pm 0.00897$ & $0.00385 \pm 0.00881$ & $y=-0.000359 x+0.00805$ & 1.00 & \\
\hline S_(36:1) & $0.999 \pm 0.437$ & $1 \pm 0.661$ & $0.824 \pm 0.375$ & $0.561 \pm 0.391$ & $y=-0.0552 x+1.27$ & 0.86 & \\
\hline S_(37:2) & $0.159 \pm 0.092$ & $0.296 \pm 0.22$ & $0.206 \pm 0.125$ & $0.08 \pm 0.0753$ & $y=-0.0121 x+0.278$ & 0.22 & \\
\hline S_(38:0) & $1.88 \pm 0.631$ & $3.39 \pm 2.13$ & $1.89 \pm 0.684$ & $1.03 \pm 0.594$ & $y=-0.15 x+3.2$ & 0.28 & \\
\hline S_(38:1-OH) & $0.153 \pm 0.242$ & $0.197 \pm 0.308$ & $0.116 \pm 0.253$ & $0.0765 \pm 0.109$ & $y=-0.0115 x+0.224$ & 0.61 & \\
\hline S_(39:1) & $0.111 \pm 0.0948$ & $0.41 \pm 0.632$ & $0.194 \pm 0.249$ & $0.0621 \pm 0.12$ & $y=-0.0134 x+0.297$ & 0.09 & \\
\hline S_(40:0) & $7.77 \pm 1.43$ & $17.9 \pm 12.7$ & $10.5 \pm 4.51$ & $7.67 \pm 4.6$ & $y=-0.285 x+13.1$ & 0.04 & \\
\hline S_(40:1-OH $)$ & $1.48 \pm 0.815$ & $1.78 \pm 1.37$ & $1.57 \pm 0.909$ & $1.46 \pm 1.39$ & $y=-0.01 x+1.65$ & 0.06 & \\
\hline S_(40:2) & $1.04 \pm 0.637$ & $1.44 \pm 0.841$ & $1.03 \pm 0.425$ & $0.566 \pm 0.349$ & $y=-0.0679 x+1.54$ & 0.44 & \\
\hline S_(41:0) & $0.352 \pm 0.195$ & $1.1 \pm 0.943$ & $0.253 \pm 0.162$ & $0.0222 \pm 0.0413$ & $y=-0.068 x+0.952$ & 0.26 & \\
\hline S_(41:2) & $\mathrm{ND} \pm \mathrm{ND}$ & $0.0108 \pm 0.0324$ & $0.021 \pm 0.0318$ & $0.0543 \pm 0.0655$ & $y=0.00806 x-0.0438$ & 0.91 & \\
\hline S_(42:0) & $0.322 \pm 0.225$ & $0.59 \pm 0.876$ & $0.491 \pm 0.223$ & $0.294 \pm 0.162$ & $y=-0.00678 x+0.476$ & 0.03 & \\
\hline S_(42:2) & $0.395 \pm 0.385$ & $1.1 \pm 1.26$ & $0.958 \pm 0.837$ & $0.983 \pm 0.763$ & $y=0.0601 x+0.399$ & 0.44 & \\
\hline S_(42:2-OH $)$ & $2.43 \pm 0.843$ & $1.55 \pm 0.588$ & $0.786 \pm 0.406$ & $0.789 \pm 0.434$ & $y=-0.211 x+3$ & 0.88 & \\
\hline S_(46:2-OH) & $0.0599 \pm 0.0337$ & $0.0149 \pm 0.0269$ & $0.0228 \pm 0.018$ & $0.0258 \pm 0.031$ & $y=-0.0035 x+0.0576$ & 0.37 & \\
\hline S_(48:2-OH) & $0.00958 \pm 0.0163$ & $\mathrm{ND} \pm \mathrm{ND}$ & $0.00395 \pm 0.00731$ & $0.00115 \pm 0.00326$ & $y=-0.00104 x+0.0133$ & 1.00 & \\
\hline SM_(30:1) & $1.61 \pm 0.262$ & $1.59 \pm 0.611$ & $1.89 \pm 0.679$ & $1.84 \pm 0.667$ & $y=0.0367 x+1.45$ & 0.68 & \\
\hline SM_(32:0) & $4.99 \pm 0.941$ & $4.66 \pm 2.13$ & $3.81 \pm 1.08$ & $2.85 \pm 1.21$ & $y=-0.269 x+6.14$ * & 0.96 & Decreasing \\
\hline SM_(32:1) & $428 \pm 88.3$ & $368 \pm 107$ & $334 \pm 87.3$ & $256 \pm 63.6$ & $y=-20.4 x+502 *$ & 0.98 & Decreasing \\
\hline SM_(33:1) & $273 \pm 37.3$ & $238 \pm 62.2$ & $168 \pm 32.2$ & $135 \pm 44.4$ & $y=-17.9 x+341 *$ & 0.98 & Decreasing \\
\hline SM_(34:0) & $21.8 \pm 5.98$ & $26 \pm 6.49$ & $22.2 \pm 6.65$ & $18 \pm 4.85$ & $y=-0.563 x+26.3$ & 0.36 & \\
\hline SM_(34:0-OH) & $7.39 \pm 2.3$ & $5.26 \pm 2.46$ & $3.5 \pm 1.7$ & $1.4 \pm 1.69$ & $y=-0.731 x+9.98 *$ & 1.00 & Decreasing \\
\hline SM_(34:1) & $3400 \pm 495$ & $3830 \pm 796$ & $3240 \pm 427$ & $2620 \pm 346$ & $y=-109 x+4100$ & 0.57 & \\
\hline SM_(34:1-OH) & $4.36 \pm 1.63$ & $10.1 \pm 8$ & $5.89 \pm 3.22$ & $2.74 \pm 2.48$ & $y=-0.336 x+8.34$ & 0.14 & \\
\hline SM_(34:2) & $397 \pm 102$ & $401 \pm 106$ & $320 \pm 50.4$ & $282 \pm 94.6$ & $y=-15.8 x+471$ & 0.88 & \\
\hline SM_(34:2-OH) & $4.44 \pm 1.37$ & $4.15 \pm 1.73$ & $3.2 \pm 0.88$ & $2.68 \pm 0.928$ & $y=-0.231 x+5.38 *$ & 0.96 & Decreasing \\
\hline SM_(35:0) & $0.957 \pm 1.35$ & $0.969 \pm 0.848$ & $0.457 \pm 0.267$ & $0.389 \pm 0.404$ & $y=-0.0821 x+1.32$ & 0.84 & \\
\hline SM_(35:1) & $36.3 \pm 3.74$ & $48.6 \pm 6.53$ & $40.9 \pm 7.5$ & $41.1 \pm 8.38$ & $y=0.248 x+39.8$ & 0.03 & \\
\hline SM_(35:2) & $0.632 \pm 0.456$ & $0.335 \pm 0.314$ & $0.144 \pm 0.149$ & $0.137 \pm 0.18$ & $y=-0.0621 x+0.787$ & 0.87 & Decreasing \\
\hline SM_(36:1) & $449 \pm 43.4$ & $529 \pm 103$ & $446 \pm 58.3$ & $298 \pm 50.5$ & $y=-19.9 x+582$ & 0.52 & \\
\hline SM_(36:2) & $32 \pm 6.24$ & $38.6 \pm 9.35$ & $30.9 \pm 4.52$ & $23.1 \pm 5.49$ & $y=-1.27 x+40.9$ & 0.49 & \\
\hline SM_(36:3) & $2.19 \pm 0.784$ & $1.71 \pm 0.891$ & $0.76 \pm 0.384$ & $0.406 \pm 0.326$ & $y=-0.233 x+3.05 *$ & 0.97 & Decreasing \\
\hline SM_(37:2) & $0.0328 \pm 0.0984$ & $0.024 \pm 0.0721$ & $\mathrm{ND} \pm \mathrm{ND}$ & $0.0197 \pm 0.0556$ & $y=-0.0015 x+0.0363$ & 0.86 & \\
\hline
\end{tabular}


Table 1. Cont

\begin{tabular}{|c|c|c|c|c|c|c|c|}
\hline & Diet 1 & Diet 2 & Diet 3 & Diet 4 & Trendline Equation & $\mathbf{R}^{2}$ & $\begin{array}{l}\text { Successive Change } \\
\text { Across Groups }\end{array}$ \\
\hline SM_(38:0) & $7.73 \pm 1.45$ & $7.83 \pm 3.24$ & $6.35 \pm 1.81$ & $4.64 \pm 1.6$ & $y=-0.398 x+9.68$ & 0.86 & \\
\hline SM_(38:1) & $342 \pm 56.4$ & $293 \pm 59.3$ & $200 \pm 27.7$ & $106 \pm 19.4$ & $y=-29.7 x+462 *$ & 0.98 & Decreasing \\
\hline SM_(39:0) & $8.55 \pm 3.43$ & $8.71 \pm 6.78$ & $12.5 \pm 7.79$ & $\mathrm{ND} \pm \mathrm{ND}$ & $\mathrm{y}=0.731 \mathrm{x}+5.31$ & 0.78 & Increasing \\
\hline SM_(39:1) & $178 \pm 28.6$ & $142 \pm 35.5$ & $109 \pm 28.1$ & $85.4 \pm 10.7$ & $y=-11.5 x+217 *$ & 0.99 & Decreasing \\
\hline SM_(40:0) & $17.3 \pm 5.88$ & $14.2 \pm 4.27$ & $10.8 \pm 2.35$ & $4.03 \pm 1.2$ & $y=-1.6 x+23.8 *$ & 0.96 & Decreasing \\
\hline SM_(40:0-OH) & $5.96 \pm 1.62$ & $4.29 \pm 1.17$ & $3.53 \pm 1.12$ & $0.919 \pm 0.868$ & $y=-0.588 x+8.17 *$ & 0.95 & Decreasing \\
\hline SM_(40:1) & $989 \pm 137$ & $867 \pm 181$ & $704 \pm 125$ & $520 \pm 71.7$ & $y=-58.1 x+1210 *$ & 0.99 & Decreasing \\
\hline SM_(40:2) & $138 \pm 21.3$ & $123 \pm 21.6$ & $91.7 \pm 16.9$ & $55.3 \pm 11.1$ & $y=-10.3 x+181 *$ & 0.97 & Decreasing \\
\hline SM_(41:0) & $8.36 \pm 2.45$ & $8.68 \pm 2.13$ & $7.03 \pm 1.56$ & $5.33 \pm 1.63$ & $y=-0.398 x+10.4$ & 0.83 & \\
\hline SM_(41:1) & $893 \pm 123$ & $839 \pm 144$ & $772 \pm 135$ & $695 \pm 133$ & $y=-24.5 x+987 *$ & 0.99 & Decreasing \\
\hline SM_(42:0) & $8.67 \pm 2.47$ & $10.5 \pm 3.94$ & $7.13 \pm 2.87$ & $3.17 \pm 1.49$ & $y=-0.736 x+13$ & 0.68 & \\
\hline SM_(42:0-OH) & $12.7 \pm 2.47$ & $11.1 \pm 1.98$ & $9.01 \pm 2.88$ & $4.92 \pm 1.53$ & $y=-0.942 x+16.6 *$ & 0.95 & Decreasing \\
\hline SM_(42:1) & $1610 \pm 185$ & $1540 \pm 227$ & $1280 \pm 206$ & $1020 \pm 194$ & $y=-75.2 x+1940 *$ & 0.95 & Decreasing \\
\hline SM_(42:2) & $1160 \pm 180$ & $1180 \pm 185$ & $1010 \pm 197$ & $880 \pm 109$ & $y=-37.4 x+1340$ & 0.86 & \\
\hline SM_(43:0) & $0.202 \pm 0.119$ & $0.527 \pm 0.275$ & $0.368 \pm 0.199$ & $0.115 \pm 0.152$ & $y=-0.0156 x+0.422$ & 0.09 & \\
\hline SM_(43:1) & $158 \pm 43.1$ & $200 \pm 35.8$ & $163 \pm 44.2$ & $153 \pm 57$ & $y=-1.93 x+183$ & 0.10 & \\
\hline SM_(44:1) & $13.5 \pm 4.24$ & $17.9 \pm 3.6$ & $14.1 \pm 4.99$ & $9.32 \pm 3.95$ & $y=-0.605 x+18.3$ & 0.36 & \\
\hline SM_(44:2) & $27 \pm 6.17$ & $29.6 \pm 5.31$ & $27 \pm 7.84$ & $23.1 \pm 5.83$ & $y=-0.53 x+30.7$ & 0.47 & \\
\hline TG_(18:0) & $11 \pm 8.91$ & $11.3 \pm 4.51$ & $6.54 \pm 4.04$ & $2.47 \pm 2.6$ & $y=-1.12 x+16.4$ & 0.88 & \\
\hline TG_(24:0) & $920 \pm 2500$ & $39.9 \pm 59.6$ & $84.9 \pm 195$ & $177 \pm 372$ & $y=-80.9 x+924$ & 0.46 & \\
\hline TG_(36:0) & $170 \pm 214$ & $52.4 \pm 36.9$ & $93.4 \pm 83.8$ & $441 \pm 288$ & $y=31.6 x-52.8$ & 0.40 & \\
\hline TG_(44:1) & $970 \pm 941$ & $325 \pm 175$ & $1550 \pm 2130$ & $2250 \pm 1250$ & $y=188 x-161$ & 0.63 & \\
\hline TG_(45:1) & $14.3 \pm 19$ & $7.72 \pm 5.87$ & $32.3 \pm 32.3$ & $83.7 \pm 49.3$ & $y=8.62 x-31.4$ & 0.76 & \\
\hline TG_(45:2) & $92.6 \pm 86.2$ & $31.6 \pm 14.8$ & $64.1 \pm 58.7$ & $84.2 \pm 53.8$ & $y=0.27 x+66.1$ & 0.00 & \\
\hline TG_(46:1) & $85.4 \pm 104$ & $22.5 \pm 16.1$ & $87.6 \pm 112$ & $269 \pm 189$ & $y=22.8 x-58.4$ & 0.56 & \\
\hline TG_(46:2) & $1060 \pm 1040$ & $293 \pm 167$ & $412 \pm 311$ & $787 \pm 609$ & $y=-25.9 x+836$ & 0.07 & \\
\hline TG_(46:4) & $3530 \pm 3550$ & $700 \pm 350$ & $464 \pm 357$ & $316 \pm 241$ & $y=-366 x+4050$ & 0.70 & Decreasing \\
\hline TG_(47:0) & $7.11 \pm 7$ & $2.59 \pm 2.23$ & $2.86 \pm 4.1$ & $6.91 \pm 2.88$ & $y=-0.0122 x+4.96$ & 0.00 & \\
\hline TG_(47:1) & $1.39 \pm 3.19$ & $0.253 \pm 0.76$ & $6.65 \pm 10.2$ & $19.6 \pm 9.01$ & $y=2.26 x-10.3$ & 0.79 & \\
\hline TG_(47:2) & $11.5 \pm 11.3$ & $3.39 \pm 2.87$ & $10.9 \pm 6.94$ & $22.3 \pm 17.7$ & $y=1.48 x+0.715$ & 0.44 & \\
\hline TG_(48:0) & $133 \pm 69.6$ & $63 \pm 10.9$ & $75.7 \pm 98.4$ & $92.4 \pm 43.9$ & $\mathrm{y}=-4.04 \mathrm{x}+122$ & 0.21 & \\
\hline TG_(48:1) & $142 \pm 100$ & $83 \pm 29.2$ & $279 \pm 343$ & $435 \pm 172$ & $y=39.8 x-69.8$ & 0.78 & \\
\hline TG_(48:2) & $241 \pm 196$ & $115 \pm 38.3$ & $319 \pm 401$ & $440 \pm 165$ & $y=29.7 x+51.8$ & 0.57 & \\
\hline TG_(48:3) & $250 \pm 185$ & $83.7 \pm 27$ & $112 \pm 80.1$ & $102 \pm 49.9$ & $y=-15.4 x+255$ & 0.49 & \\
\hline TG_(49:0) & $18 \pm 9.16$ & $10.7 \pm 2.12$ & $11 \pm 5.69$ & $10.2 \pm 3.69$ & $y=-0.856 x+19$ & 0.65 & \\
\hline TG_(49:1) & $35.7 \pm 18.6$ & $28.3 \pm 9.67$ & $67.9 \pm 55.5$ & $96.3 \pm 30.3$ & $y=8.2 x-5.68$ & 0.83 & \\
\hline TG_(49:2) & $70.2 \pm 29.8$ & $50.1 \pm 12.1$ & $77.6 \pm 55.4$ & $86.3 \pm 30.3$ & $y=2.81 x+49.6$ & 0.40 & \\
\hline TG_(49:3) & $27.3 \pm 12.9$ & $18.2 \pm 5.03$ & $26.8 \pm 15.4$ & $19.3 \pm 7.36$ & $y=-0.57 x+27.3$ & 0.17 & \\
\hline
\end{tabular}


Table 1. Cont

\begin{tabular}{|c|c|c|c|c|c|c|c|}
\hline & Diet 1 & Diet 2 & Diet 3 & Diet 4 & Trendline Equation & $\mathbf{R}^{2}$ & $\begin{array}{l}\text { Successive Change } \\
\text { Across Groups }\end{array}$ \\
\hline TG_(50:0) & $178 \pm 68.6$ & $118 \pm 20.3$ & $125 \pm 57.6$ & $102 \pm 37.6$ & $y=-8.19 x+193$ & 0.75 & \\
\hline TG $(50: 1)$ & $1440 \pm 548$ & $1030 \pm 339$ & $1870 \pm 1370$ & $1870 \pm 858$ & $y=78.9 x+949$ & 0.47 & \\
\hline TG_(50:2) & $2750 \pm 1210$ & $1830 \pm 458$ & $2920 \pm 2200$ & $2920 \pm 1010$ & $y=59.3 x+2150$ & 0.16 & \\
\hline TG_(50:3) & $1500 \pm 858$ & $1050 \pm 283$ & $1760 \pm 1370$ & $1040 \pm 452$ & $y=-24.8 x+1530$ & 0.06 & \\
\hline TG_(51:1) & $79 \pm 30.9$ & $78.4 \pm 31.8$ & $138 \pm 102$ & $124 \pm 39.6$ & $y=7.21 x+49.7$ & 0.67 & \\
\hline TG_(51:2) & $296 \pm 163$ & $274 \pm 82.5$ & $532 \pm 293$ & $509 \pm 148$ & $y=33.2 x+149$ & 0.72 & \\
\hline TG_(51:3) & $648 \pm 459$ & $467 \pm 133$ & $437 \pm 175$ & $186 \pm 86.3$ & $y=-52.4 x+836 *$ & 0.92 & Decreasing \\
\hline TG_(51:4) & $662 \pm 568$ & $330 \pm 121$ & $150 \pm 55.5$ & $37.2 \pm 17.5$ & $y=-76.1 x+877 *$ & 0.94 & Decreasing \\
\hline TG_(52:0) & $128 \pm 70.4$ & $80.4 \pm 17.6$ & $69.5 \pm 28.8$ & $47.4 \pm 24.1$ & $y=-9.36 x+153 *$ & 0.92 & Decreasing \\
\hline TG $(52: 1)$ & $849 \pm 304$ & $716 \pm 311$ & $1130 \pm 904$ & $823 \pm 275$ & $y=12.4 x+784$ & 0.06 & \\
\hline TG_(52:2) & $12,300 \pm 6460$ & $9670 \pm 3230$ & $12,700 \pm 6000$ & $8610 \pm 3300$ & $y=-298 x+13,100$ & 0.27 & \\
\hline TG_(52:3) & $36,800 \pm 25,800$ & $22,700 \pm 5870$ & $15,800 \pm 5770$ & $5160 \pm 2670$ & $y=-3770 x+49,000 *$ & 0.98 & Decreasing \\
\hline TG_(52:4) & $45,200 \pm 36,700$ & $21,100 \pm 7240$ & $9760 \pm 3330$ & $2220 \pm 1880$ & $y=-5200 x+59,300 *$ & 0.93 & Decreasing \\
\hline TG_(53:1) & $28.5 \pm 8.42$ & $23.8 \pm 10.3$ & $36.4 \pm 29.3$ & $25.5 \pm 8.57$ & $y=0.133 x+27.5$ & 0.01 & \\
\hline TG $(53: 2)$ & $253 \pm 129$ & $240 \pm 109$ & $353 \pm 259$ & $214 \pm 67.8$ & $y=-0.148 x+266$ & 0.00 & \\
\hline TG_(53:3) & $801 \pm 527$ & $518 \pm 145$ & $470 \pm 175$ & $183 \pm 73.3$ & $y=-70.4 x+1030 *$ & 0.94 & Decreasing \\
\hline TG_(53:4) & $877 \pm 722$ & $489 \pm 180$ & $277 \pm 100$ & $75.2 \pm 38.5$ & $y=-96.9 x+1170 *$ & 0.97 & Decreasing \\
\hline TG $(54: 0)$ & $20.1 \pm 12.8$ & $11.5 \pm 2.27$ & $9.51 \pm 3.73$ & $7.25 \pm 5.07$ & $y=-1.5 x+23.6$ & 0.87 & Decreasing \\
\hline TG_(54:1) & $117 \pm 54.8$ & $79 \pm 24.1$ & $96.6 \pm 86.6$ & $57.2 \pm 25.8$ & $y=-5.99 x+133$ & 0.67 & \\
\hline TG_(54:2) & $866 \pm 326$ & $637 \pm 373$ & $805 \pm 731$ & $408 \pm 146$ & $y=-44.7 x+1020$ & 0.58 & \\
\hline TG_(54:3) & $5400 \pm 3270$ & $2880 \pm 1140$ & $2350 \pm 1060$ & $1000 \pm 436$ & $y=-509 x+6800 *$ & 0.93 & Decreasing \\
\hline TG_(54:4) & $14,200 \pm 10,600$ & $6230 \pm 2180$ & $3450 \pm 1230$ & $977 \pm 444$ & $y=-1570 x+18,200 *$ & 0.91 & Decreasing \\
\hline TG $(54: 5)$ & $26,100 \pm 21,100$ & $10,200 \pm 4050$ & $4300 \pm 1350$ & $1010 \pm 595$ & $y=-3010 x+33,400$ & 0.89 & Decreasing \\
\hline TG_(54:6) & $24,900 \pm 20,300$ & $9310 \pm 3280$ & $2980 \pm 1090$ & $554 \pm 426$ & $y=-2940 x+31,900$ & 0.88 & Decreasing \\
\hline TG_(55:2) & $13.8 \pm 4.81$ & $9.88 \pm 6.38$ & $16.2 \pm 17.2$ & $8.35 \pm 3.23$ & $\mathrm{y}=-0.371 \mathrm{x}+14.9$ & 0.13 & \\
\hline TG $(55: 3)$ & $77.5 \pm 39.7$ & $46.2 \pm 18.2$ & $44.1 \pm 29.4$ & $19.7 \pm 8.29$ & $y=-6.5 x+96.6$ * & 0.91 & Decreasing \\
\hline TG_(55:4) & $142 \pm 102$ & $65.1 \pm 25.8$ & $44.9 \pm 20.2$ & $10.8 \pm 6.06$ & $y=-15.3 x+183 *$ & 0.92 & Decreasing \\
\hline TG_(55:5) & $161 \pm 137$ & $92.4 \pm 44$ & $49.8 \pm 23.4$ & $14.4 \pm 11$ & $y=-17.9 x+216^{*}$ & 0.98 & Decreasing \\
\hline TG_(55:6) & $122 \pm 143$ & $100 \pm 46.1$ & $50.3 \pm 18.8$ & $8.95 \pm 8.19$ & $y=-14.4 x+180 *$ & 0.98 & Decreasing \\
\hline TG_(56:2) & $31.6 \pm 11.6$ & $20.8 \pm 10.4$ & $30.9 \pm 40$ & $11.7 \pm 4.55$ & $y=-1.84 x+37.8$ & 0.46 & \\
\hline TG $(56: 3)$ & $189 \pm 87.4$ & $100 \pm 43.6$ & $93.3 \pm 74.8$ & $31.4 \pm 14.6$ & $y=-17.8 x+239$ & 0.91 & Decreasing \\
\hline TG_(56:6) & $6970 \pm 6060$ & $3330 \pm 1460$ & $1200 \pm 414$ & $246 \pm 173$ & $y=-826 x+9260 *$ & 0.93 & Decreasing \\
\hline TG_(56:7) & $8570 \pm 7750$ & $3690 \pm 1650$ & $1190 \pm 519$ & $282 \pm 194$ & $y=-1010 x+11,200 *$ & 0.90 & Decreasing \\
\hline TG $(56: 8)$ & $7360 \pm 7600$ & $2990 \pm 1460$ & $893 \pm 372$ & $186 \pm 119$ & $y=-875 x+9550$ & 0.89 & Decreasing \\
\hline TG_(57:2) & $0.611 \pm 0.405$ & $0.434 \pm 0.601$ & $1 \pm 1.89$ & $0.371 \pm 0.399$ & $y=-0.0057 x+0.648$ & 0.00 & \\
\hline TG_(57:6) & $83.1 \pm 100$ & $39.6 \pm 24.4$ & $16.6 \pm 3.26$ & $5.21 \pm 5.03$ & $y=-9.51 x+109^{*}$ & 0.93 & Decreasing \\
\hline TG $(58: 10)$ & $1370 \pm 1470$ & $564 \pm 268$ & $161 \pm 77.3$ & $29.9 \pm 23.1$ & $y=-164 x+1780$ & 0.90 & Decreasing \\
\hline TG_(58:7) & $1160 \pm 1150$ & $484 \pm 241$ & $123 \pm 65.2$ & $20 \pm 18.1$ & $y=-140 x+1520$ & 0.90 & Decreasing \\
\hline
\end{tabular}


Table 1. Cont.

\begin{tabular}{|c|c|c|c|c|c|c|c|}
\hline & Diet 1 & Diet 2 & Diet 3 & Diet 4 & Trendline Equation & $\mathbf{R}^{2}$ & $\begin{array}{c}\text { Successive Change } \\
\text { Across Groups }\end{array}$ \\
\hline TG_(58:8) & $1600 \pm 1440$ & $654 \pm 354$ & $186 \pm 77.9$ & $42.6 \pm 30.7$ & $y=-190 x+2080$ & 0.89 & Decreasing \\
\hline TG_(58:9) & $1730 \pm 1730$ & $730 \pm 418$ & $206 \pm 106$ & $41.2 \pm 28.3$ & $y=-207 x+2260$ & 0.90 & Decreasing \\
\hline TG_(59:3) & $0.283 \pm 0.273$ & $0.333 \pm 0.708$ & $0.539 \pm 1.26$ & $0.0277 \pm 0.0537$ & $\mathrm{y}=-0.0207 x+0.454$ & 0.12 & \\
\hline TG_(59:4) & $0.348 \pm 0.474$ & $0.22 \pm 0.449$ & $0.372 \pm 0.807$ & $0.00429 \pm 0.0121$ & $y=-0.0326 x+0.485$ & 0.45 & \\
\hline TG_(59:5) & $0.212 \pm 0.241$ & $0.104 \pm 0.128$ & $\mathrm{ND} \pm \mathrm{ND}$ & $\mathrm{ND} \pm \mathrm{ND}$ & $y=-0.04 x+0.356$ & 1.00 & \\
\hline TG_(59:6) & $0.382 \pm 0.578$ & $0.178 \pm 0.227$ & $0.137 \pm 0.174$ & $0.108 \pm 0.197$ & $y=-0.032 x+0.446$ & 0.81 & Decreasing \\
\hline TG_(59:7) & $0.214 \pm 0.434$ & $0.147 \pm 0.232$ & $\mathrm{ND} \pm \mathrm{ND}$ & $0.0326 \pm 0.0607$ & $y=-0.0222 x+0.291$ & 1.00 & \\
\hline TG_(59:8) & $9.09 \pm 14.6$ & $6.77 \pm 11.9$ & $5.83 \pm 9.99$ & $1.75 \pm 2.04$ & $y=-0.85 x+12.4 *$ & 0.94 & Decreasing \\
\hline TG_(60:10) & $194 \pm 194$ & $73.7 \pm 56.6$ & $4.21 \pm 4.15$ & $0.838 \pm 1.23$ & $y=-24 x+252$ & 0.86 & Decreasing \\
\hline TG_(60:12) & $79.5 \pm 74.4$ & $39.6 \pm 28$ & $13 \pm 12.1$ & $1.42 \pm 2.02$ & $y=-9.66 x+107^{*}$ & 0.94 & Decreasing \\
\hline TG_(62:12) & $63.3 \pm 43.7$ & $31.3 \pm 17.4$ & $11 \pm 5.05$ & $0.863 \pm 0.716$ & $y=-7.69 x+85.4 *$ & 0.95 & Decreasing \\
\hline TG_(62:13) & $4.22 \pm 2.92$ & $2.86 \pm 3.13$ & $0.546 \pm 1.15$ & $0.0336 \pm 0.0949$ & $y=-0.551 x+6.13 *$ & 0.95 & Decreasing \\
\hline
\end{tabular}




\section{Discussion}

Out of 472 lipids detected and semi-quantified, 100 showed a strong relationship with the dietary intake of ruminant fat with 35 species increasing and 65 species decreasing as the percentage of ruminant fat in the diet increased (NB. ruminant fat as a percentage composition with corn-oil and medium chain triacylglyceride oil). Interestingly, ceramides generally increased, whilst cardiolipins, sphingomyelins and triacylglycerides generally decreased as the dietary composition of ruminant fat rose. According to the literature, a rise in liver ceramides is typically associated with aggravated non-alcoholic fatty liver disease (NAFLD) and insulin resistance [23], this in conjunction with a decrease in cardiolipins (which are indicative of mitochondrial remodelling and dysfunction [24]) may suggest that the changes in the experimental diets here are detrimental for these pathologies. However, there was a clear decrease in the triacylglycerides (particularly evident in the unsaturated odd chain triacylglycerides), which is explicitly representative of an ameliorated pathology [25]. As previously published [26], many NAFLD and insulin resistance factors were mitigated as the ruminant fat increased in these diets, including: a reduction in the total body weight (g), total fat mass (\%), serum ALT (U/mL) and degree of steatosis determined by Oil Red O staining, notably, the inflammatory marker TNF $\alpha$ did not change significantly (trend: $p$-value $=0.52$ ). A key characteristic of NAFLD development is the accumulation of hepatic triacylglycerides [27]; therefore, the data here suggests that these dietary changes may be beneficial for NAFLD and insulin resistance by aiding in a reduced hepatic triacylglyceride load: possible mechanisms here include a lower saturated fatty acid composition resulting in a lower fatty acid incorporation into hepatic triacylglycerides and/or a higher pass-through of the medium chain triglyceride oil directly into the mitochondria stimulation fatty acid metabolism [26]. Work presented by Gonzalez-Cantero and colleagues [28] showed that hepatic triacylglyceride content were correlated with insulin resistance and these relationships were independently to the inflammatory marker TNF $\alpha$. Therefore, it appears that the hepatic triacylglyceride load may be paramount in the development of NAFLD and insulin resistance, which is supported in the literature [29].

According to the literature, odd chain fatty acids are considered biomarkers of their dietary intake and particularly accredited as a biomarkers of ruminant fat intake (e.g., milk, butter and beef tallow, etc.); however, there is a vast amount of conflicting data [2]. Some studies have shown both positive correlations (either individual odd chain lipids or total odd chain lipids) and some studies have shown there were no significant correlations. Although these studies may conflict in their findings they all present their data as relative compositions (Mol\%), which is the typical way lipid data appear in the literature [30]. By expressing the lipid data as relative compositions ( $\mathrm{Mol} \%$ ), it normalises the data to the total fat in that sample; however, presenting the lipid data in this way confounds the results by interconnecting the individual data points. This interconnection can cause false positive and/or false negative conclusions (type 1 and 2 errors), i.e., if a single lipid increases it will artificially decrease the other(s) due to the Mol\% calculation. As shown in the figure below (see Figure 2), the concentration of the total lipids containing either even chain or odd chain fatty acids and a combination are shown. Although lipids containing odd chain fatty acids did increase, albeit not statistically significantly; p-value: 0.197 , it was also not proportionate to the increase in dietary ruminant fat. As shown, the lipids containing even chain fatty acids did significantly inversely decrease (slope $p$-value: 0.0189 ) as the dietary ruminant fat increased. Interestingly, due to both the decrease in the even chain lipids and the consistency of the odd chain lipids, if the relative composition ( $\mathrm{Mol} \%$ ) of the lipids were calculated, there appears to be a statistically significant increase in the odd chain lipids (see Figure 3); however, this is an artefact of changes in the even chain lipids and a consequence of interconnecting the data. 


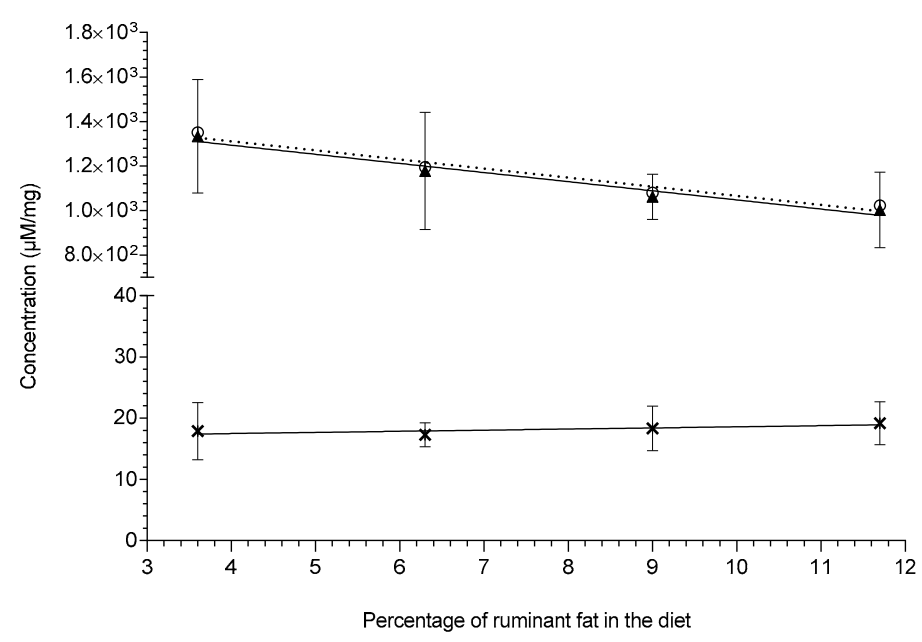

Figure 2. This figure shows the change in the liver lipid concentrations across the four high-fat diets fed to Sprague-Dawley rats ( $n=8-9$ per group): total odd chain lipids (symbol: $\mathbf{X}$, trendline: gradient: $0.183 \pm 0.0962, \mathrm{R}^{2}=0.64$, slope significance $p$-value: 0.197 ); total even chain lipids (symbol: $\boldsymbol{\Lambda}$, trendline: - , gradient: $-41.0 \pm 5.71, \mathrm{R}^{2}=0.963$, slope significance $p$-value: 0.0189 ); total lipids containing both even and of odd chain (symbol: $\mathbf{O}$, trendline: $\cdots \cdot \cdots$, gradient: $-40.8 \pm 5.79, \mathrm{R}^{2}=0.961$, slope significance $p$-value: 0.0195 . Lipid concentrations $(\mu \mathrm{M} / \mathrm{mg})$ are shown as means \pm standard deviation and were extracted via the protein precipitation liquid extraction protocol (chloroform: methanol: acetone, $\sim 7: 3: 4)$.

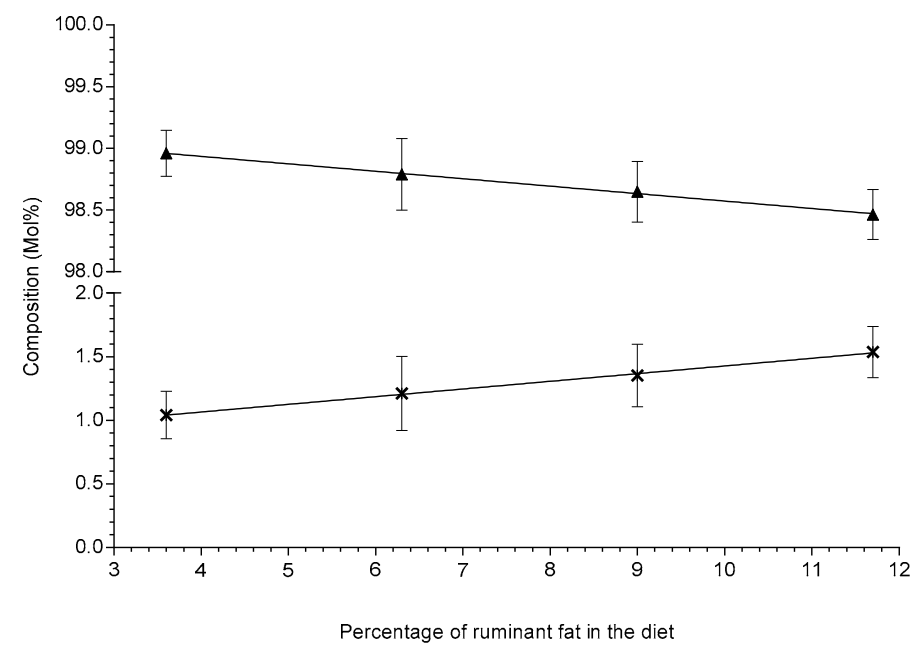

Figure 3. This figure shows the relative compositional (Mol\%) change in the total odd chain lipids (symbol: X, trendline: $\longrightarrow$, gradient: $0.0604 \pm 0.00203, \mathrm{R}^{2}=0.998$, slope significance $p$-value: 0.0011 ) and the total even chain lipids (symbol: $\boldsymbol{\Delta}$, trendline: - , gradient: $-0.0604 \pm 0.00203, R^{2}=0.998$, slope significance $p$-value: 0.0011) across the four high-fat diets in Sprague-Dawley rats $(n=8-9$ per group). Lipid compositions (Mol\%) are shown as means \pm standard deviation and were extracted via the protein precipitation liquid extraction protocol (chloroform: methanol: acetone, $7: 3: 4$ ). Diet one: $3.6 \%$ beef tallow; diet two: $6.3 \%$ beef tallow; diet three: $9.0 \%$ beef tallow; diet four: $11.7 \%$ beef tallow.

\section{Materials and Methods}

\subsection{Chemicals and Standards}

Stable isotope-labelled internal standards purchased from Sigma Aldrich (Haverhill, Suffolk, UK) include: N-palmitoyl-d31-D-erythro-sphingosine (abbreviated to IS_Cer_16:0-d31); order number: 868516P, 1-palmitoyl-d31-2-oleoyl-sn-glycero-3-phosphate (abbreviated to IS_PA_34:1-d31); order number: 860453P, 1-palmitoyl-d31-2-oleoyl-sn-glycero-3-phosphocholine (abbreviated to 
IS_PC_34:1-d31); order number: 860399P, 1-palmitoyl-d31-2-oleoyl-sn-glycero-3-phosphoethanolamine (abbreviated to IS_PE_34:1-d31); order number: 860374P,1-palmitoyl-d31-2-oleoyl-sn-glycero-3-[phospho-rac-(1-glycerol)] (abbreviated to IS_PG_34:1-d31); order number: 860384P,1-palmitoyl-d31-2-oleoyl-sn-glycero-3-phosphoinositol (abbreviated to IS_PI_34:1-d31); order number: 860042P,1,2-dimyristoyl-d54-sn-glycero-3-[phospho-L-serine] (abbreviated to IS_PS_28:0-d54); order number: 860401P, N-palmitoyl-d31-D-erythro-sphingosylphosphorylcholine (abbreviated to IS_SM_34:1-d31); order number: 868584P. Stable isotope-labelled internal standards purchased from QMX Laboratories Ltd. (QMX Laboratories Ltd., Thaxted, Essex, UK) include: Heptadecanoic-d33 acid (abbreviated to IS_FA_17:0-d33); order number: D-5261, N-tetradecylphosphocholine-d42 (abbreviated to IS_LPC_14:0-d42); order number: D-5885, Glyceryl tri(pentadecanoate-d29) (abbreviated to IS_TG_45:0-d87); order number: D-5265, Butyryl-d7-L-carnitine (abbreviated to IS_Car_4:0-d7); order number: D-7761, Hexadecanoyl-L-carnitine-d3 (abbreviated to IS_Car_16:0-d3); order number: D-6646.

Quality control standards (LIPID-QC) purchased from Cayman Chemical Company (Cambridge Bioscience, Cambridge, UK) include: Lysophosphatidylcholines (egg); order number: 24331, Phosphotidylcholines (egg); order number: 24343, Lysophosphatidylethanolamines (egg); order number: 25844, Phosphatidylethanolamines (bovine); order number: 16878, Phosphotidlethanolamine (soy); order number: 25845, Lysophosphatidyinositols (porcine liver); order number: 26016, Phosphatidylserines (soy); order number: 25847, Ceramides mixture; order number: 22853, Ceramides (non-hydroxy); order number: 24833, Ceramides (hydroxy); order number: 24834, Sphingomyelins (from bovine spinal cord); order number: 22674, Sphingomylins (egg); order number: 24345, Phosphatidylglycerols (egg); order number: 25846, Phosphatidic acid (egg); order number: 24344, Sulfatides (bovine); order number: 24323, Purified mixed gangliosides (bovine); order number: 24856, TLC Neutral Glycosphingolipid Mixture (bovine and porcine); order number: 1505, 2-Palmitoyl Glycerol; order number: CAY17882, 1,2-Dipalmitoyl-sn-glycerol; order number: CAY10008648. Quality control standards purchased from Sigma Aldrich include: Soy PC (95\%); order number: 441601G, C18(Plasm)-18:1-PC; order number: 852467C, Brain CPE; order number: 860066P, Liver PI; order number: 840042P, Brain lyso PS; order number: 850092P, Milk SM Sphingomyelin (Milk, Bovine); order number: 860063P, Galactocerebrosides from bovine brain; order number: C4905, Glucosylceramide (Soy); order number: 131304P, Triglyceride mix, C2-C10; order number: 17810-1amp-s, Fish oil from menhaden; order number: F8020, Anhydrous butter fat, Cardiolipin solution from bovine heart; order number: C1649, Brain PI(4)P; order number: 840045P.

Commercially available blank human serum was purchased from BioIVT (Royston, Hertfordshire, UK; order number: HUMANSRMPNN. All solvents and additives were of HPLC grade or higher and purchased from Sigma Aldrich unless otherwise stated.

LIPID-IS: the lipid stable isotope-labelled internal standard was prepared by dissolving each of the individual lipid standards into chloroform: methanol (1:1) solution to produce a $1 \mathrm{mM}$ primary stock solution. From each of these stock solutions, $1 \mathrm{~mL}$ was transferred into a volumetric flask and diluted with methanol to reach a final working solution concentration of $5 \mu \mathrm{M}$ in methanol of IS_Cer_16:0-d31, IS_FA_17:0-d33, IS_LPC_14:0-d42, IS_PA_34:1-d31, IS_PC_34:1-d31, IS_PE_34:1-d31, IS_PG_34:1-d31, IS_PI_34:1-d31, IS_PS_28:0-d54, IS_SM_34:1-d31, IS_TG_45:0-d87.

ACYL-CARNITINE-IS: the acyl-carnitine stable isotope-labelled internal standard was prepared by dissolving each powdered stock into methanol to achieve a $5 \mathrm{mM}$ stock solution. Taking $1 \mathrm{~mL}$ of the IS_Car_4:0-d7 and IS_Car_16:0-d3 stock solutions and diluting these into methanol until a final working solution of $5 \mu \mathrm{M}$ was achieved for IS_Car_4:0-d7 and IS_Car_16:0-d3.

LIPID-QC: the lipid quality control standards were prepared by diluting each lipid mix to achieve a $50 \mu \mathrm{g} / \mathrm{mL}$ working stock solution in propan-2-ol: acetonitrile: water (2:1:1, respectively).

\subsection{Extraction}

Lipids were isolated comparing two methods; firstly, a novel protein-precipitation liquid extraction and secondly the liquid-liquid extraction previously described by Folch and colleagues [12] in an 
adapted version as we described previously [31]. Tissue quantities ranged from 2-50 $\mathrm{mg}$ and fluid samples from 10-50 $\mu \mathrm{L}$ (e.g., plasma/serum) were tested (data not shown here).

\subsubsection{Protein Precipitation Liquid Extraction Protocol (PPLE)}

The protein-precipitation liquid extraction protocol was as follows: the tissue samples were weighed (NB. fluid samples were pipetted) and transferred into a $2 \mathrm{~mL}$ screw cap Eppendorf plastic tube (Eppendorf, Stevenage, UK) along with a single $5 \mathrm{~mm}$ stainless steel ball bearing. Immediately, $400 \mu \mathrm{L}$ of chloroform: methanol (2:1, respectively) solution was added to each sample, followed by thorough mixing. The samples were then homogenised in the chloroform: methanol (2:1, respectively) using a Bioprep 24-1004 homogenizer (Allsheng, Hangzhou, China) run at speed; $4.5 \mathrm{~m} / \mathrm{s}$, time; $30 \mathrm{~s}$ for 2 cycles. Then, $400 \mu \mathrm{L}$ of chloroform, $100 \mu \mathrm{L}$ of the LIPID-IS (5 $\mu \mathrm{M}$ in methanol) and $100 \mu \mathrm{L}$ of the CARNITINE-IS ( $5 \mu \mathrm{M}$ in methanol) was added to each sample. The samples were homogenised again using a Bioprep 24-1004 homogenizer run at speed; $4.5 \mathrm{~m} / \mathrm{s}$, time; $30 \mathrm{~s}$ for 2 cycles. To ensure fibrous material was diminished, the samples were sonicated for $30 \mathrm{~min}$ in a water bath sonicator (Advantage-Lab, Menen, Belgium). Then, $400 \mu \mathrm{L}$ of acetone was added to each sample. The samples were thoroughly vortexed and centrifuged for $10 \mathrm{~min}$ at $\sim 20,000 \times \mathrm{g}$ to pellet any insoluble material at the bottom of the vial. The single layer supernatant was pipetted into separate $2 \mathrm{~mL}$ screw cap amber-glass auto-sampler vials (Agilent Technologies, Cheadle, UK); being careful not to break up the solid pellet at the bottom of the tube. The organic extracts (chloroform, methanol, acetone composition, $\sim 1.4 \mathrm{~mL}$ ) were dried down to dryness using a Concentrator Plus system (Eppendorf, Stevenage, UK) run for $60 \mathrm{~min}$ at $60^{\circ} \mathrm{C}$. The samples were reconstituted in $100 \mu \mathrm{L}$ of 2:1:1 (propan-2-ol, acetonitrile and water, respectively) then thoroughly vortex. The reconstituted sample was transferred into a $250 \mu \mathrm{L}$ low-volume vial insert inside a $2 \mathrm{~mL}$ amber glass auto-sample vial ready for liquid chromatography with mass spectrometry detection (LC-MS) analysis.

\subsubsection{Folch Liquid-Liquid Extraction Protocol (Folch LLE)}

The Folch liquid-liquid extraction protocol is as follows: the tissue samples were weighed (NB. fluid samples were pipetted) and transferred into a $2 \mathrm{~mL}$ screw cap Eppendorf plastic tube (Eppendorf, Stevenage, UK) along with a single $5 \mathrm{~mm}$ stainless steel ball bearing. Immediately, $400 \mu \mathrm{L}$ of chloroform: methanol (2:1, respectively) solution was added to each sample, followed by thorough mixing. The samples were then homogenised in the chloroform: methanol (2:1, respectively) using a Bioprep 24-1004 homogenizer (Allsheng, Hangzhou, China) run at speed; $4.5 \mathrm{~m} / \mathrm{s}$, time; $30 \mathrm{~s}$ for 2 cycles. Then, $400 \mu \mathrm{L}$ of chloroform, $100 \mu \mathrm{L}$ of the LIPID-IS ( $5 \mu \mathrm{M}$ in methanol) and $100 \mu \mathrm{L}$ of the ACYL-CARNITINE-IS ( $5 \mu \mathrm{M}$ in methanol) was added to each sample. The samples were homogenised again using a Bioprep 24-1004 homogenizer run at speed; $4.5 \mathrm{~m} / \mathrm{s}$, time; $30 \mathrm{~s}$ for 2 cycles. To ensure fibrous material was diminished, the samples were sonicated for $30 \mathrm{~min}$ in a water bath sonicator. Then, $400 \mu \mathrm{L}$ of HPLC water was added to each samples. The samples were thoroughly vortexed and centrifuged for $10 \mathrm{~min}$ at $\sim 20,000 \mathrm{~g}$ to separate the two immiscible fractions. The organic fractions (the lower layer, mostly chloroform; $\sim 700 \mu \mathrm{L}$ ) and aqueous fractions (the upper layer, methanol and water; $\sim 700 \mu \mathrm{L}$ ) were pipetted into separate $2 \mathrm{~mL}$ screw cap amber-glass auto-sampler vials (Agilent Technologies, Cheadle, UK); being careful not to break up the solid pellet between the layers. To ensure complete lipid isolation a double extraction protocol was followed; $1 \mathrm{~mL}$ of chloroform: methanol (2:1, respectively) solution was added to each sample, along with $400 \mu \mathrm{L}$ of HPLC water. The samples were thoroughly vortexed and centrifuged for $10 \mathrm{~min}$ at $\sim 20,000 \times g$. The organic fractions and aqueous fractions were pipetted into the corresponding $2 \mathrm{~mL}$ screw cap amber-glass auto-sampler vials containing the initial extracts (again being careful not to break up the solid pellet between the layers). The combined organic extracts $(\sim 1.4 \mathrm{~mL})$ were dried down to dryness using a Concentrator Plus system (Eppendorf, Stevenage, UK) run for $60 \mathrm{~min}$ at $60^{\circ} \mathrm{C}$. The samples were reconstituted in $100 \mu \mathrm{L}$ of 2:1:1 (propan-2-ol, acetonitrile and water, respectively) then thoroughly vortex. The reconstituted sample 
was transferred into a $250 \mu \mathrm{L}$ low-volume vial insert inside a $2 \mathrm{~mL}$ amber glass auto-sample vial ready for liquid chromatography with mass spectrometry detection (LC-MS) lipidomics analysis.

\subsection{LC-MS Method}

Full chromatographic separation of intact lipids was achieved using a Shimadzu HPLC System (Shimadzu UK Limited, Milton Keynes, UK) with the injection of $10 \mu \mathrm{L}$ onto a Waters Acquity UPLC ${ }^{\circledR}$ CSH C18 column (Waters, Hertfordshire, UK); $1.7 \mu \mathrm{m}$, I.D. $2.1 \mathrm{~mm} \times 50 \mathrm{~mm}$, maintained at $55^{\circ} \mathrm{C}$. Mobile phase A was 6:4, acetonitrile and water with $10 \mathrm{mM}$ ammonium formate. Mobile phase B was 9:1, propan-2-ol and acetonitrile with $10 \mathrm{mM}$ ammonium formate. The flow was maintained at $500 \mu \mathrm{L}$ per minute through the following gradient: $0.00 \mathrm{~min} \_40 \%$ mobile phase $\mathrm{B} ; 0.40 \mathrm{~min} \_43 \%$ mobile phase B; 0.45 min_50\% mobile phase B; 2.40 min_54\% mobile phase B; 2.45 min_70\% mobile phase $B ; 7.00$ min_ $99 \%$ mobile phase $B ; 8.00$ min_99\% mobile phase B; 8.3 min_40\% mobile phase B; 10 min $\_40 \%$ mobile phase B. The sample injection needle was washed using 9:1, 2-propan-2-ol and acetonitrile. The mass spectrometer used was the Thermo Scientific Exactive Orbitrap with a heated electrospray ionisation source (Thermo Fisher Scientific, Hemel Hempstead, UK). The mass spectrometer was calibrated immediately before sample analysis using positive and negative ionisation calibration solution (recommended by Thermo Scientific). Additionally, the heated electrospray ionisation source was optimised at 50:50 mobile phase A to mobile phase B for spray stability (capillary temperature; $300{ }^{\circ} \mathrm{C}$, source heater temperature; $420^{\circ} \mathrm{C}$, sheath gas flow; 40 (arbitrary), auxiliary gas flow; 15 (arbitrary), spare gas; 3 (arbitrary), source voltage; $4 \mathrm{kV}$. The mass spectrometer scan rate set at $4 \mathrm{~Hz}$, giving a resolution of 25,000 (at $200 \mathrm{~m} / \mathrm{z}$ ) with a full-scan range of $\mathrm{m} / \mathrm{z} 100$ to 1800 with continuous switching between positive and negative mode.

\subsection{Data Processing}

Thermo Xcalibur Quan Browser (Thermo Fisher Scientific, Hemel Hempstead, UK) data processing involved the integration of the internal standard extracted ion chromatogram (EIC) peaks at the expected retention times (see Table 2). The EIC were selected from the ionisation mode for each analyte class; the ionisation mode is dependent on the molecular chemistry of the analytes, i.e., basic chemical groups ordinarily result in positive ionisation (e.g., $\left.[\mathrm{M}+\mathrm{H}]^{+}, \mathrm{M}+\mathrm{H}-\mathrm{H}_{2} \mathrm{O}\right]^{+},[\mathrm{M}+\mathrm{Na}]^{+},\left[\mathrm{M}+\mathrm{NH}_{4}\right]^{+},[\mathrm{M}+\mathrm{K}]^{+}$) whereas acidic chemical groups typically result in negative ionisation (e.g., $[\mathrm{M}-\mathrm{H}]^{-}$).

Table 2. This table shows the stable isotope-labelled internal standards with their ionisation products (i.e., $\left.[\mathrm{M}+\mathrm{H}]^{+}, \mathrm{M}+\mathrm{H}-\mathrm{H}_{2} \mathrm{O}\right]^{+},[\mathrm{M}+\mathrm{Na}]^{+},\left[\mathrm{M}+\mathrm{NH}_{4}\right]^{+},[\mathrm{M}+\mathrm{K}]^{+},[\mathrm{M}-\mathrm{H}]^{-}$) and primary ionisation mode (positive; +ve or negative; -ve), along with their retention time (minutes). Butyryl-d7-L-carnitine (abbreviated to IS_Car_4:0-d7), N-tetradecylphosphocholine-d42 (abbreviated to IS_LPC_14:0-d42), hexadecanoyl-L-carnitine-d3 (abbreviated to IS_Car_16:0-d3), heptadecanoic-d33 acid (abbreviated to IS_FA_17:0-d33), 1,2-dimyristoyl-d54-sn-glycero-3-[phospho-L-serine] (abbreviated to IS_PS_28:0-d54), 1-palmitoyl-d31-2-oleoyl-sn-glycero-3-phosphoinositol (abbreviated to IS_PI_34:1-d31), N-palmitoyl-d31-D-erythro-sphingosylphosphorylcholine (abbreviated to IS_SM_34:1-d31), 1-palmitoyl-d31-2-oleoyl-sn-glycero-3-[phospho-rac-(1-glycerol)] (abbreviated to IS_PG_34:1-d31), 1-palmitoyl-d31-2-oleoyl-sn-glycero-3-phosphate (abbreviated to IS_PA_34:1-d31), N-palmitoyl-d31-D-erythro-sphingosine (abbreviated to IS_Cer_16:0-d31), 1-palmitoyl-d31-2-oleoyl-sn-glycero-3-phosphocholine (abbreviated to IS_PC_34:1-d31), 1-palmitoyl-d31-2-oleoyl-sn-glycero-3-phosphoethanolamine (abbreviated to IS_PE_34:1-d31), glyceryl tri(pentadecanoate-d29) (abbreviated to IS_TG_45:0-d87).

\begin{tabular}{cccc}
\hline Internal Standard & Ionisation Product $(\mathrm{m} / \mathrm{z})$ & $\begin{array}{c}\text { Ionisation } \\
\text { Mode }\end{array}$ & $\begin{array}{c}\text { Expected Retention } \\
\text { Time (mins) }\end{array}$ \\
\hline IS_Car_4:0-d7 & 239.1983 & $+\mathrm{ve}$ & 0.3 \\
IS_LPC_14:0-d42 & $422.5560,421.5498,420.5435$ & $+\mathrm{ve}$ & 0.4 \\
IS_Car_16:0-d3 & 403.3610 & $+\mathrm{ve}$ & 0.5 \\
IS_FA_17:0-d33 & $302.4557,301.4495,300.4432$ & $-\mathrm{ve}$ & 1.1 \\
IS_PS_28:0-d54 & $732.7741,731.7678,730.7615,729.7553,728.7490$ & $-\mathrm{ve}$ & 1.4 \\
\hline
\end{tabular}


Table 2. Cont.

\begin{tabular}{|c|c|c|c|}
\hline Internal Standard & Ionisation Product $(\mathrm{m} / \mathrm{z})$ & $\begin{array}{c}\text { Ionisation } \\
\text { Mode }\end{array}$ & $\begin{array}{l}\text { Expected Retention } \\
\text { Time (mins) }\end{array}$ \\
\hline IS_PI_34:1-d31 & $864.7162,865.7225,866.7288$ & - ve & 2.9 \\
\hline IS_SM_34:1-d31 & $733.7632,734.7670,755.7451,756.7514,771.7190,772.7253$ & + ve & 3.0 \\
\hline IS_PG_34:1-d31 & $775.6939,776.7002,777.7065,778.7127$ & - ve & 3.0 \\
\hline IS_PA_34:1-d31 & $700.6509,701.6571,702.6634,703.6697,704.6760$ & - ve & 3.4 \\
\hline IS_Cer_16:0-d31 & $\begin{array}{l}548.6851,549.6914,550.6977,551.7039,566.6951,567.7014 \\
568.7076,569.7139,590.6896,591.6959,606.6636,607.6698\end{array}$ & + ve & 3.9 \\
\hline IS_PC_34:1-d31 & $790.7700,791.7750,812.7553,813.7616,828.7292,829.7355$ & + ve & 3.9 \\
\hline IS_PE_34:1-d31 & $\begin{array}{c}\text { 747.7181, } 748.7254,749.7327,769.7021,770.7084,771.7146, \\
785.6760,786.6823,787.6886\end{array}$ & +ve & 4.0 \\
\hline IS_TG_45:0-d87 & $\begin{array}{c}850.2239,851.2301,852.2364,853.2427,867.2504,868.2567 \\
869.2630,870.2693,872.2059,873.2121,874.2184,875.2247 \\
888.1798,889.1861,890.1923,891.1986\end{array}$ & + ve & 5.8 \\
\hline
\end{tabular}

As shown in the table above (see Table 2), the internal standards have multiple ionisation products, these are the result of numerous ionisation mechanism (for example IS_TG_45:0-d87 having different adducts: $[\mathrm{M}+\mathrm{H}]^{+},[\mathrm{M}+\mathrm{Na}]^{+},[\mathrm{M}+\mathrm{K}]^{+}$and $\left[\mathrm{M}+\mathrm{NH}_{4}\right]^{+}$, present) as well as an isotopic distribution (e.g., IS_TG_45:0-d87 having either the expected eighty-seven or fewer deuterium atoms present) all reasonably expected ions were included into the EIC for each internal standard (see Figure 4).

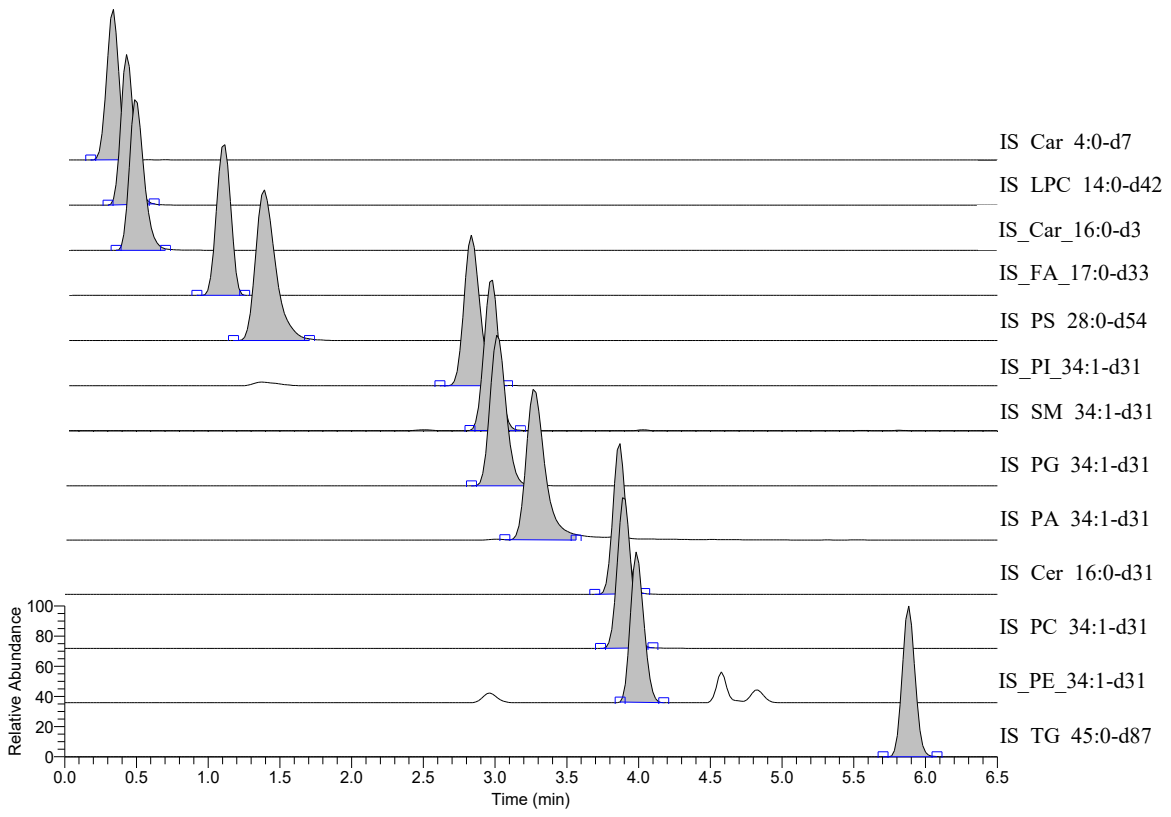

Figure 4. This figure shows a spiked (5 $\mu \mathrm{M}$ in methanol) commercial human plasma extracted ion chromatogram (EIC) for the stable isotope-labelled internal standards (lipids and acyl-carnitines): butyryl-d7-L-carnitine (abbreviated to IS_Car_4:0-d7); area $\sim 5.9 \times 10^{6}$ counts, N-tetradecylphosphocholine-d42 (abbreviated to IS_LPC_14:0-d42); are $\sim 2.1 \times 10^{8}$ counts, hexadecanoyl-L-carnitine-d3 (abbreviated to IS_Car_16:0-d3); area $\sim 5.8 \times 10^{8}$ counts, heptadecanoic-d33 acid (abbreviated to IS_FA_17:0-d33); area 1.1 × 104 counts, 1,2-dimyristoyl-d54sn-glycero-3-[phospho-L-serine] (abbreviated to IS_PS_28:0-d54); area $\sim 2.2 \times 10^{7}$ counts, 1-palmitoyl-d31-2-oleoyl-sn-glycero-3-phosphoinositol (abbreviated to IS_PI_34:1-d31): area $\sim 1.1 \times 10^{7}$ counts, N-palmitoyl-d31-D-erythro-sphingosylphosphorylcholine (abbreviated to IS_SM_34:1-d31); $1.4 \times 10^{8}$ counts, 1-palmitoyl-d31-2-oleoyl-sn-glycero-3-[phospho-rac-(1-glycerol)] (abbreviated to IS_PG_34:1-d31); area $\sim 6.1 \times 10^{7}$ counts, 1-palmitoyl-d31-2-oleoyl-sn-glycero-3-phosphate (abbreviated to IS_PA_34:1-d31); area $\sim 1.3 \times 10^{7}$ counts, N-palmitoyl-d31-D-erythro-sphingosine (abbreviated to IS_Cer_16:0-d31); area $\sim 2.2 \times 10^{8}$ counts, 1-palmitoyl-d31-2-oleoyl-sn-glycero-3phosphocholine (abbreviated to IS_PC_34:1-d31); area $2.5 \times 10^{8}$ counts, 1-palmitoyl-d31-2-oleoylsn-glycero-3-phosphoethanolamine (abbreviated to IS_PE_34:1-d31); area $\sim 6.3 \times 10^{7}$ counts, glyceryl tri(pentadecanoate-d29) (abbreviated to IS_TG_45:0-d87); area $\sim 3.2 \times 10^{7}$ counts. 
The adduct composition of the total EIC produced from each of the ionisation mechanisms are shown in the figure below (see Figure 5).

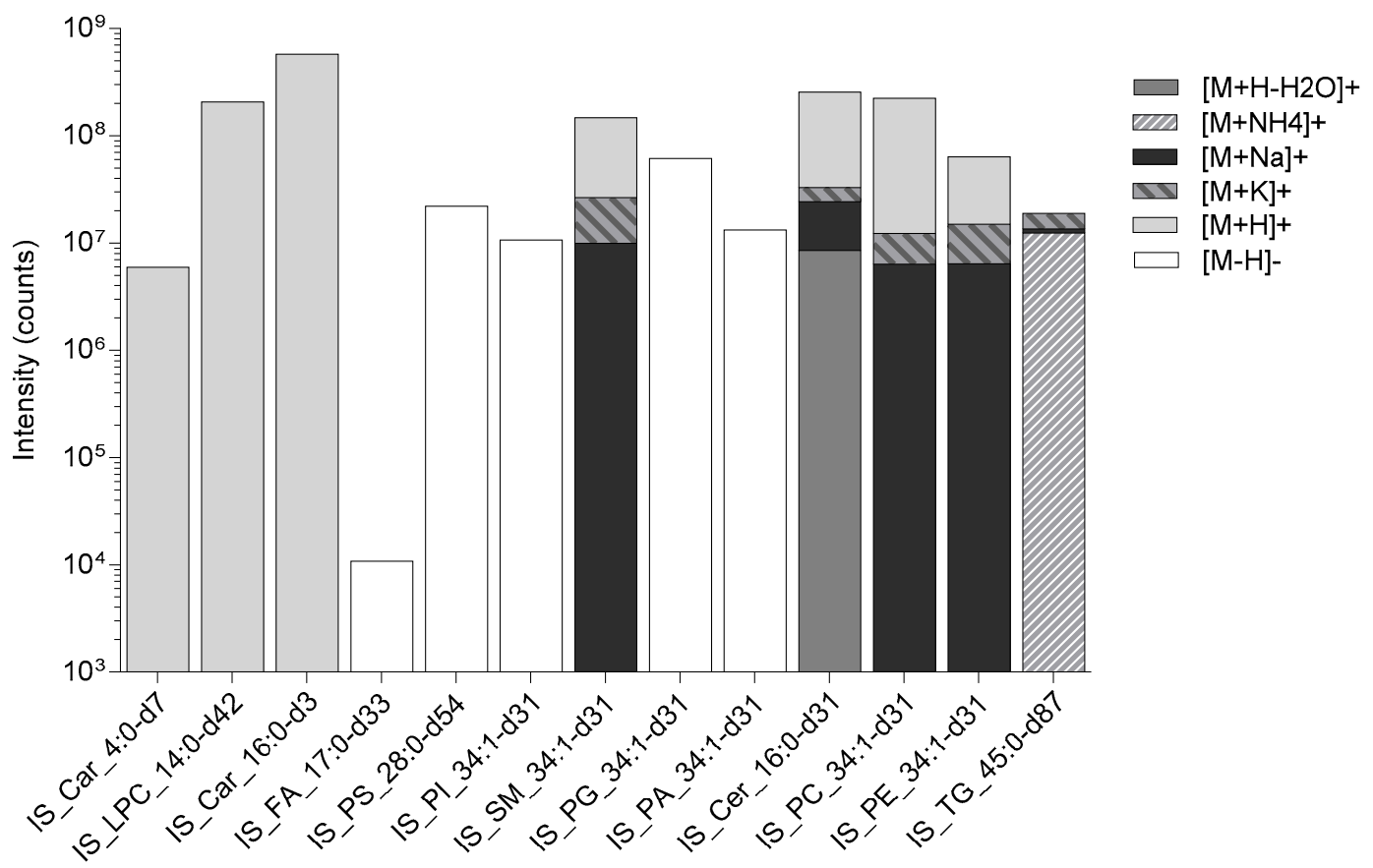

Figure 5. This figure shows the intensity of the extracted ion chromatogram for each stable isotope-labelled internal standard along with the ionisation adduct composition: $[\mathrm{M}+\mathrm{H}]^{+},\left[\mathrm{M}+\mathrm{H}-\mathrm{H}_{2} \mathrm{O}\right]^{+},\left[\mathrm{M}+\mathrm{NH}_{4}\right]^{+},[\mathrm{M}+\mathrm{Na}]^{+},[\mathrm{M}+\mathrm{K}]^{+}$and $[\mathrm{M}-\mathrm{H}]^{-}$. Butyryl-d7-L-carnitine (abbreviated to IS_Car_4:0-d7), N-tetradecylphosphocholine-d42 (abbreviated to IS_LPC_14:0-d42), hexadecanoyl-L-carnitine-d3 (abbreviated to IS_Car_16:0-d3), heptadecanoic-d33 acid (abbreviated to IS_FA_17:0-d33), 1,2-dimyristoyl-d54-sn-glycero-3-[phospho-L-serine] (abbreviated to IS_PS_28:0-d54), 1-palmitoyl-d31-2-oleoyl-sn-glycero-3-phosphoinositol (abbreviated to IS_PI_34:1-d31)， N-palmitoyl-d31-D-erythro-sphingosylphosphorylcholine (abbreviated to IS_SM_34:1-d31), 1-palmitoyl-d31-2-oleoyl-sn-glycero-3-[phospho-rac-(1-glycerol)] (abbreviated to IS_PG_34:1-d31), 1-palmitoyl-d31-2-oleoyl-sn-glycero-3-phosphate (abbreviated to IS_PA_34:1-d31), N-palmitoyl-d31-D-erythro-sphingosine (abbreviated to IS_Cer_16:0-d31), 1-palmitoyl-d31-2-oleoyl-sn-glycero-3-phosphocholine (abbreviated to IS_PC_34:1-d31), 1-palmitoyl-d31-2-oleoyl-sn-glycero-3-phosphoethanolamine (abbreviated to IS_PE_34:1-d31), glyceryl tri(pentadecanoate-d29) (abbreviated to IS_TG_45:0-d87).

The data processing also involved the integration of the individual lipid (and derivatives) species at their expected retention time (see Supplementary Table S2) allowing for a maximum of $\pm 0.1 \mathrm{~min}$ of retention time drift: any retention time drift greater than $\pm 0.1 \mathrm{~min}$ resulted in the exclusion of the analyte leading to a 'Not Found' result (i.e., zero concentration). A list of the analyte classes along with the number of species detected within each class are shown in the table below (see Table 3). The expected adducts for each analyte class and the internal standard used for semi-quantitation are also shown.

The lipid quality control (QC) standards were analysed with each batch of samples, these QC standards were used to check the retention times for the analytes ensuring that isobaric analytes were separated and expected analyte retention times remained robust.

Through the Thermo Xcalibur Quan Browser software, the responses of the analytes were normalised to the relevant internal standard response (producing area ratios) (see Supplementary Table S2), these area ratios corrected the intensity for any extraction and instrument variations. The area ratios were then blank corrected where intensities less than three times the blank samples were set to 
a 'Not Found' result (i.e., zero concentration). The accepted area ratios were then multiplied by the concentration of the internal standard to give the analyte concentrations. The results for fluid samples were expressed in molar concentrations (typically $\mu \mathrm{M}$ or $\mathrm{nM}$ ). For tissue samples, the calculated concentrations of the analytes were then divided by the amount of tissue (in $\mathrm{mg}$ ) used in the extraction protocol to give the final results in $\mu \mathrm{M}$ per $\mathrm{mg}$ of tissue extracted $(\mu \mathrm{M} / \mathrm{mg})$.

Table 3. This table shows the lipid classes detected with this LC-MS lipidomics method. The number of species per lipid class and the measured adducts (protonated: $[\mathrm{M}+\mathrm{H}]^{+}$, deprotonated: $[\mathrm{M}-\mathrm{H}]^{-}$, protonated with water loss: $\left[\mathrm{M}+\mathrm{H}-\mathrm{H}_{2} \mathrm{O}\right]^{+}$, sodiated: $[\mathrm{M}+\mathrm{Na}]^{+}$, potasiated: $[\mathrm{M}+\mathrm{K}]^{+}$, ammoniated: $\left[\mathrm{M}+\mathrm{NH}_{4}\right]^{+}$) are also shown. The internal standard used for semi-quantification are also shown: butyryl-d7-L-carnitine (abbreviated to IS_Car_4:0-d7), N-tetradecylphosphocholine-d42 (abbreviated to IS_LPC_14:0-d42), hexadecanoyl-L-carnitine-d3 (abbreviated to IS_Car_16:0-d3), heptadecanoic-d33 acid (abbreviated to IS_FA_17:0-d33), 1,2-dimyristoyl-d54-sn-glycero-3-[phospho-L-serine] (abbreviated to IS_PS_28:0-d54), 1-palmitoyl-d31-2-oleoyl-sn-glycero-3-phosphoinositol (abbreviated to IS_PI_34:1-d31), N-palmitoyl-d31-D-erythro-sphingosylphosphorylcholine (abbreviated to IS_SM_34:1-d31), 1-palmitoyl-d31-2-oleoyl-sn-glycero-3-[phospho-rac-(1-glycerol)] (abbreviated to IS_PG_34:1-d31), 1-palmitoyl-d31-2-oleoyl-sn-glycero-3-phosphate (abbreviated to IS_PA_34:1-d31), N-palmitoyl-d31-D-erythro-sphingosine (abbreviated to IS_Cer_16:0-d31), 1-palmitoyl-d31-2-oleoyl-sn-glycero-3-phosphocholine (abbreviated to IS_PC_34:1-d31), 1-palmitoyl-d31-2-oleoyl-sn-glycero-3-phosphoethanolamine (abbreviated to IS_PE_34:1-d31), glyceryl tri(pentadecanoate-d29) (abbreviated to IS_TG_45:0-d87).

\begin{tabular}{|c|c|c|c|}
\hline Analyte Class & No. of Species & Adducts & Internal Standard \\
\hline Acyl-carnitines & 48 & {$[\mathrm{M}+\mathrm{H}]^{+}$} & $\begin{array}{l}\text { IS_Car_4:0-d7, } \\
\text { IS_Car_16:0-d3 }\end{array}$ \\
\hline Ceramides & 85 & {$[\mathrm{M}+\mathrm{H}]^{+},\left[\mathrm{M}+\mathrm{H}-\mathrm{H}_{2} \mathrm{O}\right]^{+}$} & IS_Cer_16:0-d31 \\
\hline Cardiolipins & 56 & {$[\mathrm{M}-\mathrm{H}]^{-}$} & IS_TG_45:0-d87 \\
\hline Diacylglycerols & 6 & {$\left[\mathrm{M}+\mathrm{H}-\mathrm{H}_{2} \mathrm{O}\right]^{+},[\mathrm{M}+\mathrm{Na}]^{+},[\mathrm{M}+\mathrm{K}]^{+}$} & IS_TG_45:0-d87 \\
\hline Gangliosides (GM1) & 24 & {$[\mathrm{M}-\mathrm{H}]^{-}$} & IS_PG_34:1-d31 \\
\hline Hexosylceramides & 56 & {$[\mathrm{M}+\mathrm{H}]^{+},\left[\mathrm{M}+\mathrm{H}-\mathrm{H}_{2} \mathrm{O}\right]^{+}$} & IS_Cer_16:0-d31 \\
\hline Lyso-phosphatidylcholines & 23 & {$[\mathrm{M}+\mathrm{H}]^{+}$} & IS_LPC_14:0-d42 \\
\hline Lyso-phosphatidyethanolamines & 19 & {$[\mathrm{M}+\mathrm{H}]^{+}$} & IS_LPC_14:0-d42 \\
\hline Lyso-phosphatidylinositols & 19 & {$[\mathrm{M}-\mathrm{H}]^{-}$} & IS_PI_34:1-d31 \\
\hline Lyso-phosphoserines & 20 & {$[\mathrm{M}-\mathrm{H}]^{-}$} & IS_PS_28:0-d54 \\
\hline Lyso-cardiolipins & 23 & {$[\mathrm{M}-\mathrm{H}]^{-}$} & IS_TG_45:0-d87 \\
\hline Monoacylglycerols & 1 & {$\left[\mathrm{M}+\mathrm{H}-\mathrm{H}_{2} \mathrm{O}\right]^{+},[\mathrm{M}+\mathrm{Na}]^{+},[\mathrm{M}+\mathrm{K}]^{+}$} & IS_TG_45:0-d87 \\
\hline Phosphatidic acids & 26 & {$[\mathrm{M}-\mathrm{H}]^{-}$} & IS_PA_34:1-d31 \\
\hline Phosphatidylcholines & 43 & {$[\mathrm{M}+\mathrm{H}]^{+}$} & IS_PC_34:1-d31 \\
\hline Phosphatidylethanolamines & 19 & {$[\mathrm{M}+\mathrm{H}]^{+}$} & IS_PE_34:1-d31 \\
\hline Phosphatidylglycerol & 34 & {$[\mathrm{M}-\mathrm{H}]^{-}$} & IS_PG_34:1-d31 \\
\hline Phosphatidylinositols & 21 & {$[\mathrm{M}-\mathrm{H}]^{-}$} & IS_PI_34:1-d31 \\
\hline Phosphatidylserines & 36 & {$[\mathrm{M}-\mathrm{H}]^{-}$} & IS_PS_28:0-d54 \\
\hline Sulfatides & 72 & {$[\mathrm{M}-\mathrm{H}]^{-}$} & IS_PG_34:1-d31 \\
\hline Sphingomyelins & 54 & {$[\mathrm{M}+\mathrm{H}]^{+},[\mathrm{M}+\mathrm{Na}]^{+},[\mathrm{M}+\mathrm{K}]^{+}$} & IS_SM_34:1-d31 \\
\hline Triacylglycerides & 89 & {$[\mathrm{M}+\mathrm{H}]^{+},\left[\mathrm{M}+\mathrm{NH}_{4}\right]^{+},[\mathrm{M}+\mathrm{Na}]^{+},[\mathrm{M}+\mathrm{K}]^{+}$} & IS_TG_45:0-d87 \\
\hline
\end{tabular}

\subsection{Animal Intervention}

Sprague-Dawley rats (Harlan, IN, USA) were overfed using one of four experimental diets ( $n=6-9$ per group) at $17 \%$ above matched growth via an intragastric cannula surgically inserted as previously described [26]. Animals had ad libitum access to water throughout the experiments. The four experimental diets were $70 \%$ fat (\% energy) including different amounts of medium chain triacylglycerides oil (MCT), beef tallow and corn oil; the fat composition of each diet are shown in the table below (see Table 4).

Protein (19\% whey protein), vitamin and mineral contents were the same in all diets. Diets were formulated to meet the caloric and nutritional recommendations established by the National Research Council (NRC), but were fed at a level that exceeded the recommended caloric intake by $17 \%$ to increase weight gain and adiposity and produce steatohepatitis. 
Liver tissue was collected after 21 days. All experimental procedures were ethically approved by the Institutional Animal Care and Use Committee at the University of Arkansas for Medical Science.

Table 4. This table shows the dietary fat composition of each of the four experimental diets fed to Sprague-Dawley rats ( $n=8-9$ per group). MCT: medium chain triglyceride oil.

\begin{tabular}{cccc}
\hline Diet & Corn Oil & MCT Oil & Beef Tallow \\
\hline 1 & $50 \%$ & $16.4 \%$ & $3.6 \%$ \\
2 & $35 \%$ & $28.7 \%$ & $6.3 \%$ \\
3 & $20 \%$ & $41.0 \%$ & $9.0 \%$ \\
4 & $5 \%$ & $53.3 \%$ & $11.7 \%$ \\
\hline
\end{tabular}

\section{Conclusions}

This lipidomics protocol has been developed to quantify lipids across a broad range of hydrophobicities, from acyl-carnitines through to long chain glycerolipids. The extraction method produces a single liquid supernatant phase ideal for high-throughput workflows with an increased extraction capability over the frequently published liquid-liquid extraction previously published by Folch and colleagues [12].

Following the establishment and validation of this method, we applied it to a ruminant fat dose response dietary intervention in Sprague-Dawley rats, where we found 100 lipid species correlated strongly with the composition of ruminant fat within the diet.

It has been previously suggested that dietary ruminant fat is beneficial/protective in type 2 diabetes [32], the results presented in this manuscript suggest possible target mechanisms that need to be examined could include ceramide fatty acid compositions, cardiolipin remodeling, sphingomyelins and/or triacylglycerides concentration (particularly unsaturated odd chain species) and their associated fatty acid compositions, as well as the liver total lipid content.

Supplementary Materials: The following are available online at http://www.mdpi.com/2218-1989/10/7/296/s1, Figure S1: This figure shows the comparison between the two lipid extraction techniques regarding their extraction efficiency on each lipid class detected in the rat liver samples (Folch liquid-liquid extraction with a compositions of chloroform: methanol: water, $7: 3: 4$, and Protein precipitation liquid extraction with a composition of chloroform: methanol: acetone, $7: 3: 4) . n=34$ rat liver samples per extraction method. The intensity of the lipids were measured by liquid chromatography with mass spectrometry. The significance of the difference between the two extraction protocols are shown by the $p$-value star system; where $p \leq 0.05$ was considered statistically significant $\left({ }^{*} p<0.05\right.$, $\left.{ }_{* *}^{*} p<0.01,{ }^{* * *} p<0.001\right)$. Error bars represent \pm standard deviation. Table S1: This table shows the comparison between the two lipid extraction techniques regarding their extraction efficiency on the total intensity of each lipid class detected in the rat liver samples by each sample extraction method (Folch-LLE: Folch liquid-liquid extraction with a compositions of chloroform: methanol: water, 7:3:4, and PPLE: protein precipitation liquid extraction with a composition of chloroform: methanol: acetone, $\sim 7: 3: 4) . n=34$ rat liver samples per extraction method. The percentage increase the PPLE method is over the Folch-LLE method is shown (\% diff.) along with the $p$-value resulting from a $t$-test $(p<0.05$ designates statistical significance are in bold \& shaded). The total number of lipid species detected and pass the quality control process are also shown. Table S2: This table shows the lipids quantified in this LC-MS method, along with the ionisation mode (either positive; +ve, or negative; -ve), the detected ion $(\mathrm{m} / z)$, the expected retention time (minutes) and the internal standard used for normalisation and quantification. Lipid are shown in their shorthand notations with the number of carbons and unsaturated bonds in the fatty acid moiety separated by a colon; acyl-carnitines (Carn), ceramides (Cer), cardiolipins (CL), diacylglycerols (DG), gangliosides (GM1), hexosylceramides (Hex-Cer), lyso-phosphatidylcholines (LPC), lyso-phosphatidyethanolamines (LPE), lyso-phosphatidylinositols (LPI), lyso-cardiolipins (Lyso_CL), phosphatidic acids (PA), phosphatidylcholines (PC), phosphatidylethanolamines (PE), phosphatidylglycerol (PG), phosphatidylinositols (PI), phosphatidylserines (PS), sulfatides (S), sphingomyelins (SM), triacylglycerides (TG).

Author Contributions: Conceptualization, B.J.; methodology, B.J.; validation, B.J.; formal analysis, B.J.; investigation, B.J. and M.R.; resources, M.R. and A.K.; data curation, B.J.; writing-original draft preparation, B.J., M.R. and A.K.; writing—review and editing, B.J., M.R. and A.K.; visualization, B.J.; supervision, M.R. and A.K.; project administration, B.J.; funding acquisition, M.R. and A.K. All authors have read and agreed to the published version of the manuscript.

Funding: This research was funded by BBSRC, grant number BB/M027252/1, USDA, grant number ACNC-USDA-CRIS 6251-51000-005-03S, and COST Action, grant number CM0603. 
Conflicts of Interest: The authors declare no conflict of interest.

\section{References}

1. $\quad$ LIPID MAPS ${ }^{\circledR}$ Lipidomics Gateway LIPID MAPS ${ }^{\circledR}$ Lipidomics Gateway. Available online: https://www. lipidmaps.org/ (accessed on 20 April 2020).

2. Jenkins, B.; West, J.A.; Koulman, A. A Review of Odd-Chain Fatty Acid Metabolism and the Role of Pentadecanoic Acid (C15:0) and Heptadecanoic Acid (C17:0) in Health and Disease. Molecules 2015, 20, 2425-2444. [CrossRef] [PubMed]

3. Berg, J.M.; Tymoczko, J.L.; Stryer, L. Biochemistry, 5th ed.; W.H. Freeman: New York, NY, USA, 2002.

4. Nakamura, M.T.; Yudell, B.E.; Loor, J.J. Regulation of energy metabolism by long-chain fatty acids. Progress Lipid Res. 2014, 53, 124-144. [CrossRef] [PubMed]

5. Wymann, M.P.; Schneiter, R. Lipid signalling in disease. Nat. Rev. Mol. Cell Biol. 2008, 9, 162-176. [CrossRef] [PubMed]

6. Harayama, T.; Riezman, H. Understanding the diversity of membrane lipid composition. Nat. Rev. Mol. Cell Biol. 2018, 19, 281-296. [CrossRef]

7. Jenkins, B.J.; Seyssel, K.; Chiu, S.; Pan, P.-H.; Lin, S.-Y.; Stanley, E.; Ament, Z.; West, J.A.; Summerhill, K.; Griffin, J.L.; et al. Odd Chain Fatty Acids; New Insights of the Relationship Between the Gut Microbiota, Dietary Intake, Biosynthesis and Glucose Intolerance. Sci. Rep. 2017, 7, 44845. [CrossRef]

8. Jenkins, B.; de Schryver, E.; Van Veldhoven, P.P.; Koulman, A. Peroxisomal 2-Hydroxyacyl-CoA Lyase Is Involved in Endogenous Biosynthesis of Heptadecanoic Acid. Molecules 2017, 22, 1718. [CrossRef]

9. Zarrouk, A.; Debbabi, M.; Bezine, M.; Karym, E.M.; Badreddine, A.; Rouaud, O.; Moreau, T.; Cherkaoui-Malki, M.; El Ayeb, M.; Nasser, B.; et al. Lipid Biomarkers in Alzheimer's Disease. Curr. Alzheimer Res. 2018, 15, 303-312. [CrossRef]

10. Dorcely, B.; Katz, K.; Jagannathan, R.; Chiang, S.S.; Oluwadare, B.; Goldberg, I.J.; Bergman, M. Novel biomarkers for prediabetes, diabetes, and associated complications. Diabetes Metab. Syndr. Obes. 2017, 10, 345-361. [CrossRef]

11. Niki, E. Biomarkers of lipid peroxidation in clinical material. Biochim. Biophys. Acta (BBA) Gen. Subj. 2014, 1840, 809-817. [CrossRef]

12. Folch, J.; Lees, M.; Stanley, G.H.S. A Simple Method for the Isolation and Purification of Total Lipides from Animal Tissues. J. Biol. Chem. 1957, 226, 497-509.

13. Bligh, E.G.; Dyer, W.J. A Rapid Method of Total Lipid Extraction and Purification. Can. J. Biochem. Physiol. 1959, 37, 911-917. [CrossRef]

14. Matyash, V.; Liebisch, G.; Kurzchalia, T.V.; Shevchenko, A.; Schwudke, D. Lipid extraction by methyl-tert-butyl ether for high-throughput lipidomics. J. Lipid Res. 2008, 49, 1137-1146. [CrossRef] [PubMed]

15. Gil, A.; Zhang, W.; Wolters, J.C.; Permentier, H.; Boer, T.; Horvatovich, P.; Heiner-Fokkema, M.R.; Reijngoud, D.-J.; Bischoff, R. One- vs two-phase extraction: Re-evaluation of sample preparation procedures for untargeted lipidomics in plasma samples. Anal. Bioanal. Chem. 2018, 410, 5859-5870. [CrossRef] [PubMed]

16. Pellegrino, R.M.; Di Veroli, A.; Valeri, A.; Goracci, L.; Cruciani, G. LC/MS lipid profiling from human serum: A new method for global lipid extraction. Anal. Bioanal. Chem. 2014, 406, 7937-7948. [CrossRef] [PubMed]

17. Cajka, T.; Fiehn, O. Comprehensive analysis of lipids in biological systems by liquid chromatography-mass spectrometry. Trends Anal. Chem. 2014, 61, 192-206. [CrossRef]

18. Cajka, T.; Fiehn, O. Increasing lipidomic coverage by selecting optimal mobile-phase modifiers in LC-MS of blood plasma. Metabolomics 2016, 12, 34. [CrossRef]

19. Isaac, G.; McDonald, S.; Astarita, G. Lipid Separation Using UPLC with Charged Surface Hybrid Technology; Waters Corp: Milford, MA, USA, 2011; pp. 1-8.

20. Ulmer, C.Z.; Patterson, R.E.; Koelmel, J.P.; Garrett, T.J.; Yost, R.A. A Robust Lipidomics Workflow for Mammalian Cells, Plasma, and Tissue Using Liquid-Chromatography High- Resolution Tandem Mass Spectrometry. Methods Mol. Biol. 2017, 1609, 91-106. [CrossRef]

21. Haider, A.; Wei, Y.-C.; Lim, K.; Barbosa, A.D.; Liu, C.-H.; Weber, U.; Mlodzik, M.; Oras, K.; Collier, S.; Hussain, M.M.; et al. PCYT1A Regulates Phosphatidylcholine Homeostasis from the Inner Nuclear Membrane in Response to Membrane Stored Curvature Elastic Stress. Dev. Cell 2018, 45, 481-495.e8. [CrossRef] 
22. Petkevicius, K.; Virtue, S.; Bidault, G.; Jenkins, B.; Çubuk, C.; Morgantini, C.; Aouadi, M.; Dopazo, J.; Serlie, M.; Koulman, A.; et al. Accelerated phosphatidylcholine turnover in macrophages promotes adipose tissue inflammation in obesity. eLife 2019, 8, e47990. [CrossRef]

23. Pagadala, M.; Kasumov, T.; McCullough, A.J.; Zein, N.N.; Kirwan, J.P. Role of ceramides in nonalcoholic fatty liver disease. Trends Endocrinol. Metab. 2012, 23, 365-371. [CrossRef]

24. Shi, Y. Emerging roles of cardiolipin remodeling in mitochondrial dysfunction associated with diabetes, obesity, and cardiovascular diseases. J. Biomed. Res. 2010, 24, 6-15. [CrossRef]

25. Kawano, Y.; Cohen, D.E. Mechanisms of hepatic triglyceride accumulation in non-alcoholic fatty liver disease. J. Gastroenterol. 2013, 48, 434-441. [CrossRef] [PubMed]

26. Ronis, M.J.J.; Baumgardner, J.N.; Sharma, N.; Vantrease, J.; Ferguson, M.; Tong, Y.; Wu, X.; Cleves, M.A.; Badger, T.M. Medium chain triglycerides dose-dependently prevent liver pathology in a rat model of non-alcoholic fatty liver disease. Exp. Biol. Med. 2013, 238, 151-162. [CrossRef] [PubMed]

27. Alkhouri, N.; Dixon, L.J.; Feldstein, A.E. Lipotoxicity in Nonalcoholic Fatty Liver Disease: Not All Lipids Are Created Equal. Expert Rev. Gastroenterol. Hepatol. 2009, 3, 445-451. [CrossRef] [PubMed]

28. Gonzalez-Cantero, J.; Martin-Rodriguez, J.L.; Gonzalez-Cantero, A.; Arrebola, J.P.; Gonzalez-Calvin, J.L. Insulin resistance in lean and overweight non-diabetic Caucasian adults: Study of its relationship with liver triglyceride content, waist circumference and BMI. PLoS ONE 2018, 13, e192663. [CrossRef]

29. Dongiovanni, P.; Stender, S.; Pietrelli, A.; Mancina, R.M.; Cespiati, A.; Petta, S.; Pelusi, S.; Pingitore, P.; Badiali, S.; Maggioni, M.; et al. Causal relationship of hepatic fat with liver damage and insulin resistance in nonalcoholic fatty liver. J. Intern. Med. 2018, 283, 356-370. [CrossRef]

30. Liebisch, G.; Ahrends, R.; Arita, M.; Arita, M.; Bowden, J.A.; Ejsing, C.S.; Griffiths, W.J.; Holčapek, M.; Köfeler, H.; Mitchell, T.W.; et al. Lipidomics needs more standardization. Nat. Metab. 2019, 1, 745-747. [CrossRef]

31. Virtue, S.; Petkevicius, K.; Moreno-Navarrete, J.M.; Jenkins, B.; Hart, D.; Dale, M.; Koulman, A.; Fernández-Real, J.M.; Vidal-Puig, A. Peroxisome Proliferator-Activated Receptor $\gamma 2$ Controls the Rate of Adipose Tissue Lipid Storage and Determines Metabolic Flexibility. Cell Rep. 2018, 24, 2005-2012.e7. [CrossRef]

32. Forouhi, N.G.; Koulman, A.; Sharp, S.J.; Imamura, F.; Kröger, J.; Schulze, M.B.; Crowe, F.L.; Huerta, J.M.; Guevara, M.; Beulens, J.W.; et al. Differences in the prospective association between individual plasma phospholipid saturated fatty acids and incident type 2 diabetes: The EPIC-InterAct case-cohort study. Lancet Diabetes Endocrinol. 2014, 2, 810-818. [CrossRef] 